\title{
EXISTENCE AND UNIQUENESS OF MAXIMAL STRONG SOLUTION OF A 1D BLOOD FLOW IN A NETWORK OF VESSELS
}

\author{
DEBAYAN MAITY, JEAN-PIERRE RAYMOND, AND ARNAB ROY
}

\begin{abstract}
We study the well-posedness of a system of one-dimensional partial differential equations modeling blood flows in a network of vessels with viscoelastic walls. We prove the existence and uniqueness of maximal strong solution for this type of hyperbolic/parabolic model. We also prove a stability estimate under suitable nonlinear Robin boundary conditions.
\end{abstract}

\section{INTRODUCTION}

In this paper, we consider a one dimensional blood flow model in a network of vessels with viscoelastic walls. In each vessel (of length 1 in nondimensional variables), the cross sectional area $A(x, t)$ of the vessel at the axial coordinate $x \in I=(0,1)$ and at time $t>0$, the flow rate $Q(x, t)$, and the average internal pressure $P(x, t)$, over a cross section, satisfy the mass conservation and momentum balance equations:

$$
\begin{aligned}
& \frac{\partial A}{\partial t}+\frac{\partial Q}{\partial x}=0, \quad x \in I, t>0, \\
& \frac{\partial Q}{\partial t}+\frac{\partial}{\partial x}\left(\frac{Q^{2}}{A}\right)+\frac{A}{\rho} \frac{\partial P}{\partial x}=-k_{f} \frac{Q}{A}, \quad x \in I, t>0,
\end{aligned}
$$

where $\rho$ is the fluid density, assumed to be constant, and $k_{f}$ is the friction coefficient per unit length. To close the system we need a constitutive law connecting the pressure $P$ to the cross-sectional area $A$. In the Kelvin-Voigt model, the pressure law (or vessel law) is given by:

$$
P=P_{\text {ext }}+\frac{\beta}{A_{0}}\left(\sqrt{A}-\sqrt{A_{0}}\right)+\frac{\nu}{A_{0}} \frac{\partial}{\partial t}(\sqrt{A})
$$

where $P_{\text {ext }}$ denotes the constant external pressure, $A_{0}$ denotes the reference cross-sectional area, $\nu$ is a viscoelastic coefficient depending on the thickness $h$ of the vessel, the coefficient $\beta$ is related to the vessel stiffness, and is defined by $\beta=\frac{\sqrt{\pi} h E}{1-\sigma^{2}}$, where $E$ is the Young's modulus and $\sigma$ is the Poisson's ratio. The system (1.1)-(1.2) has to be completed by initial and boundary conditions.

For simplicity, we analyze models corresponding to the vessel law (1.2), but the results of the paper can be adapted to more general vessel laws as those considered in [17].

When we substitute the pressure law $(1.2)$ in $(1.1)_{2}$, by taking $(1.1)_{1}$ into account, we obtain the following system

$$
\begin{aligned}
& \frac{\partial A}{\partial t}+\frac{\partial Q}{\partial x}=0, \quad x \in I, t>0, \\
& \frac{\partial Q}{\partial t}+\frac{\partial}{\partial x}\left(\frac{Q^{2}}{A}\right)+\frac{\beta \sqrt{A}}{2 A_{0} \rho} \frac{\partial A}{\partial x}+\frac{\nu}{4 A_{0} A^{1 / 2} \rho} \frac{\partial A_{i}}{\partial x} \frac{\partial Q}{\partial x}-\frac{\nu \sqrt{A}}{2 A_{0} \rho} \frac{\partial^{2} Q}{\partial x^{2}}=-k_{f} \frac{Q}{A}, \quad x \in I, t>0 .
\end{aligned}
$$

Date: August 5, 2020.

2010 Mathematics Subject Classification. 35M10, 35Q35, 76D03, 76Z05, 92C35.

Key words and phrases. Blood flow model, viscoelastic vessels, one-dimensional model, arterial network, strong solutions, maximal-in-time solutions, uniqueness of solution, fluid-structure interaction.

The first author was partially supported by Department of Atomic Energy, Government of India, under project no. 12-R \& D-TFR-5.01-0520 and by the European Research Council (ERC) under the European Union's Horizon 2020 research and innovation programme (grant agreement No 694126-DYCON). The second author is partially supported by the ANR-Project HANUMAN 18CE45-0014-01. The third author is supported by the Czech Science Foundation (GAČR) project GA19-04243S. The Institute of Mathematics, CAS is supported by RVO:67985840. 
When the viscoelastic coefficient $\nu$ is equal to zero, the system corresponding to (1.3) can be written as a quasilinear hyperbolic system (see, e.g., $[6,18,23,10,26]$ ). The diffusive effect, induced by the viscous term $-\frac{\nu \sqrt{A}}{2 A_{0} \rho} \frac{\partial^{2} Q}{\partial x^{2}}$ when $\nu>0$, makes the system of hyperbolic/parabolic nature. Even if, in blood flow models, the hyperbolic nature of system (1.3) is dominent, because the viscous term $-\frac{\nu \sqrt{A}}{2 A_{0} \rho} \frac{\partial^{2} Q}{\partial x^{2}}$ is small compared to the other terms, this additional viscous term plays a role in numerical simulations [19], in estimation problems $[7,14]$, and when data coming from numerical models are compared with in vivo data [3, 4].

The viscoelastic behavior of vessels has been observed in several experimental studies [1, 27]. Several studies demonstrate that the incorporation of viscoleastic tube laws allows more physiological predictions than those obtained with elastic laws, because blood pressure and vessel deformation are often overestimated by $1 \mathrm{D}$ elastic models [22, 21, 25]. For the analysis of other viscoelastic models we refer to [20, 22, 7].

In the numerical approximations of system (1.3), the viscous term is often considered as a viscous correction in a quasilinear hyperbolic system, and therefore the viscous term is taken into account as a source term [18, 19]. Splitting methods are other numerical strategies, consisting of solving alternatively an hyperbolic system and a parabolic equation, see [17, 24].

From the well-posedness point of view, the existence of global-in-time regular solutions under some smallness conditions, or local-in-time regular solutions, for the quasilinear hyperbolic system corresponding to $\nu=0$, is studied in [6] and in [10] in a single vessel. The coupling of a quasilinear hyperbolic system with a Windkessel type boundary condition is considered in [9]. As far as we know, similar results in the viscous case, when $\nu>0$, are not known. Another viscoelastic model is derived in [5] for a single vessel, but not for a network.

The goal of this paper is to prove the existence and uniqueness of maximal strong solution of a system modeling a blood flow in a network of vessels, corresponding to (1.3) in each vessel. In this paper, due to its length, we do not study outflow boundary conditions of Windkessel type. But several results of the present paper may be extended to such models. This will be studied in a forthcoming paper.

Before studying a general network, for clarity, we introduce the first results for a binary vascular bifurcation as represented in Figure 1. For $i=1,2,3,\left(A_{i}, Q_{i}\right)$ satisfies (1.1) with the pressure law defined in (1.2). More precisely, we consider the following system:

$$
\begin{aligned}
& \text { For } i \in\{1,2,3\},\left(A_{i}, Q_{i}\right) \text { satisfies } \\
& \frac{\partial A_{i}}{\partial t}+\frac{\partial Q_{i}}{\partial x}=0, \quad t \in(0, T), x \in I, \\
& \frac{\partial Q_{i}}{\partial t}+\frac{\partial}{\partial x}\left(\frac{Q_{i}^{2}}{A_{i}}\right)+\frac{A_{i}}{\rho} \frac{\partial P_{i}}{\partial x}=-k_{f} \frac{Q_{i}}{A_{i}}, \quad t \in(0, T), x \in I, \\
& P_{i}=P_{\text {ext }}+\frac{\beta}{A_{i, 0}}\left(\sqrt{A_{i}}-\sqrt{A_{i, 0}}\right)+\frac{\nu}{A_{i, 0}} \frac{\partial}{\partial t}\left(\sqrt{A_{i}}\right),
\end{aligned}
$$

where $A_{i, 0}$ denotes the reference sectional area of the $i$-th vessel.

At the branching point, the balance of rate flows and the continuity of total pressures read as follows

$$
\begin{aligned}
& Q_{1}(1, t)=Q_{2}(0, t)+Q_{3}(0, t), \quad t \geqslant 0, \\
& P_{1}(1, t)+\frac{\rho}{2} \frac{Q_{1}^{2}}{A_{1}^{2}}(1, t)=P_{2}(0, t)+\frac{\rho}{2} \frac{Q_{2}^{2}}{A_{2}^{2}}(0, t)=P_{3}(0, t)+\frac{\rho}{2} \frac{Q_{3}^{2}}{A_{3}^{2}}(0, t), \quad t \geqslant 0 .
\end{aligned}
$$

The above system is completed by the following initial conditions

$$
A_{i}(x, 0)=A_{i}^{0}(x), \quad Q_{i}(x, 0)=Q_{i}^{0}(x), \quad x \in I,
$$

and boundary conditions

$$
Q_{1}(0, t)=h_{1}(t), \quad Q_{2}(1, t)=h_{2}(t), \quad Q_{3}(1, t)=h_{3}(t), \quad t \geqslant 0 .
$$

Using the expression of $P_{i}$ in $(1.4)_{3}$, we want to eliminate all the terms involving $P_{i}$. Observe that using (1.4) 1 , we can rewrite $P_{i}$ as

$$
P_{i}=P_{\text {ext }}+\frac{\beta}{A_{i, 0}}\left(\sqrt{A_{i}}-\sqrt{A_{i, 0}}\right)-\frac{\nu}{2 A_{i, 0} \sqrt{A_{i}}} \frac{\partial Q_{i}}{\partial x} .
$$




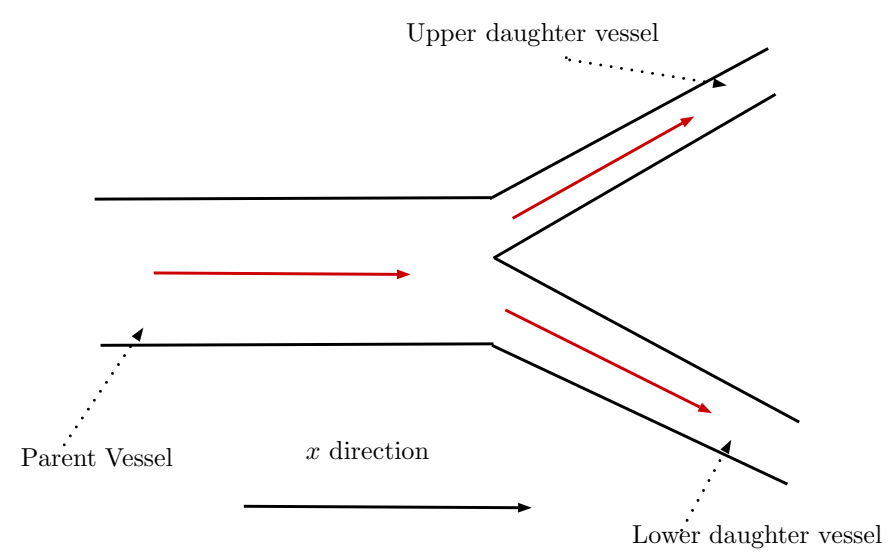

FIGURE 1

By differentiating the above pressure law with respect to $x$, we obtain

$$
\frac{\partial P_{i}}{\partial x}=\frac{\beta}{2 A_{i, 0} \sqrt{A_{i}}} \frac{\partial A_{i}}{\partial x}-\frac{\partial}{\partial x}\left(\frac{\nu}{2 A_{i, 0} \sqrt{A_{i}}} \frac{\partial Q_{i}}{\partial x}\right) .
$$

Using the above expression, we can write the system (1.4)-(1.7), in an arbitrary time interval $\left(t_{0}, t_{1}\right)$, in the following form:

$$
\left\{\begin{array}{l}
\text { For } i \in\{1,2,3\},\left(A_{i}, Q_{i}\right) \text { satisfies } \\
\frac{\partial A_{i}}{\partial t}+\frac{\partial Q_{i}}{\partial x}=0, \quad t \in\left(t_{0}, t_{1}\right), x \in I, \\
\frac{\rho}{A_{i}} \frac{\partial Q_{i}}{\partial t}+\frac{\rho}{A_{i}}\left(\frac{2 Q_{i}}{A_{i}} \frac{\partial Q_{i}}{\partial x}-\frac{Q_{i}^{2}}{A_{i}^{2}} \frac{\partial A_{i}}{\partial x}\right)+\frac{\beta}{2 A_{i, 0} \sqrt{A_{i}}} \frac{\partial A_{i}}{\partial x}-\frac{\partial}{\partial x}\left(\frac{\nu}{2 A_{i, 0} \sqrt{A_{i}}} \frac{\partial Q_{i}}{\partial x}\right) \\
=-k_{f} \rho \frac{Q_{i}}{A_{i}^{2}}, \quad t \in\left(t_{0}, t_{1}\right), x \in I, \\
Q_{1}(1, t)=Q_{2}(0, t)+Q_{3}(0, t), \quad t \in\left(t_{0}, t_{1}\right), \\
Q_{1}(0, t)=h_{1}(t), \quad Q_{2}(1, t)=h_{2}(t), \quad Q_{3}(1, t)=h_{3}(t), \quad t \in\left(t_{0}, t_{1}\right), \\
{\left.\left[-\frac{\nu}{2 A_{1,0} \sqrt{A_{1}}} \frac{\partial Q_{1}}{\partial x}+\frac{\beta}{A_{1,0}}\left(\sqrt{A_{1}}-\sqrt{A_{1,0}}\right)+\frac{1}{2} \rho \frac{Q_{1}^{2}}{A_{1}^{2}}\right]\right|_{x=1}} \\
=\left.\left[-\frac{\nu}{2 A_{2,0} \sqrt{A_{2}}} \frac{\partial Q_{2}}{\partial x}+\frac{\beta}{A_{2,0}}\left(\sqrt{A_{2}}-\sqrt{A_{2,0}}\right)+\frac{1}{2} \rho \frac{Q_{2}^{2}}{A_{2}^{2}}\right]\right|_{x=0} \\
\quad=-\left.\left[\frac{\nu}{2 A_{3,0} \sqrt{A_{3}}} \frac{\partial Q_{3}}{\partial x}+\frac{\beta}{A_{3,0}}\left(\sqrt{A_{3}}-\sqrt{A_{3,0}}\right)+\frac{1}{2} \rho \frac{Q_{3}^{2}}{A_{3}^{2}}\right]\right|_{x=0}, \quad t \in\left(t_{0}, t_{1}\right), \\
A_{i}\left(x, t_{0}\right)=A_{i}^{0}(x), \quad Q_{i}\left(x, t_{0}\right)=Q_{i}^{0}(x), \quad x \in I .
\end{array}\right.
$$

We shall need to consider system (1.8) over a time interval $\left(t_{0}, t_{1}\right)$, and not necessarily over a fixed time interval $(0, T)$, to prove the existence of maximal strong solutions (see Section 5.1).

From now on, to simplify the notation we set

$$
A=\left(A_{i}\right)_{i=1}^{3}, A^{0}=\left(A_{i}^{0}\right)_{i=1}^{3}, \bar{A}=\left(\bar{A}_{i}\right)_{i=1}^{3}, Q=\left(Q_{i}\right)_{i=1}^{3}, Q^{0}=\left(Q_{i}^{0}\right)_{i=1}^{3},(A, Q)=\left(A_{i}, Q_{i}\right)_{i=1}^{3} .
$$

For $-\infty<t_{0}<t_{1}<\infty$, we look for solutions to system (1.8) in the space

$$
\mathcal{E}\left(t_{0}, t_{1}\right)=\left\{(A, Q) \mid A \in\left[H^{1}\left(t_{0}, t_{1} ; H^{1}(I)\right)\right]^{3}, Q \in\left[L^{2}\left(t_{0}, t_{1} ; H^{2}(I)\right) \cap H^{1}\left(t_{0}, t_{1} ; L^{2}(I)\right)\right]^{3}\right\},
$$


equipped with the norm

$$
\begin{gathered}
\|(A, Q)\|_{\mathcal{E}\left(t_{0}, t_{1}\right)}=\sum_{i=1}^{3}\left(\left\|A_{i}\right\|_{H^{1}\left(t_{0}, t_{1} ; H^{1}(0,1)\right)}+\left\|A_{i}\right\|_{L^{\infty}\left(t_{0}, t_{1} ; H^{1}(0,1)\right)}+\left\|Q_{i}\right\|_{L^{2}\left(t_{0}, t_{1} ; H^{2}(0,1)\right)}\right. \\
\left.+\left\|Q_{i}\right\|_{H^{1}\left(t_{0}, t_{1} ; L^{2}(0,1)\right)}+\left\|Q_{i}\right\|_{L^{\infty}\left(t_{0}, t_{1} ; H^{1}(0,1)\right)}\right) .
\end{gathered}
$$

Moreover, for $\bar{A} \in\left[H^{1}(I)\right]^{3}, A \in\left[H^{1}\left(t_{0}, t_{1} ; H^{1}(I)\right)\right]^{3}$, we introduce the following quantities

$$
\begin{aligned}
& \gamma_{\bar{A}}=\min \{\bar{A}(x) \mid x \in \bar{I}\}>0, \\
& \gamma_{A}\left(t_{0}, t_{1}\right)=\min \left\{A(x, t) \mid x \in \bar{I}, t \in\left[t_{0}, t_{1}\right]\right\}>0 .
\end{aligned}
$$

We also introduce the spaces

$$
E_{\bar{A}}\left(t_{0}, t_{1}\right)=\left\{A \in\left[H^{1}\left(t_{0}, t_{1} ; H^{1}(I)\right)\right]^{3} \mid A\left(t_{0}\right)=\bar{A}\right\},
$$

and, for $\gamma>0$,

$$
E_{\bar{A}}\left(t_{0}, t_{1} ; \gamma\right)=\left\{A \in E_{\bar{A}}\left(t_{0}, t_{1}\right) \mid \gamma_{A}\left(t_{0}, t_{1}\right) \geqslant \gamma\right\}
$$

Definition 1.1. We say that a pair $(A, Q)$ is a strong solution to system (1.8) over the time interval $[0, T]$ when $(A, Q) \in \mathcal{E}(0, T), \gamma_{A}(0, T)>0,(A, Q)$ satisfies $(1.8)_{2-4}$ in the sense of distributions in $I \times(0, T)$ and $(1.8)_{5-9}$ in the sense of traces.

We say that $(A, Q)$ is a maximal strong solution to system (1.8) over the time interval $\left[0, T_{\mathrm{m}}\right)$ when either $T_{\mathrm{m}}=\infty$, or $T_{\mathrm{m}}<\infty$ and, for all $0<T<T_{\mathrm{m}},(A, Q)$ is a strong solution to system (1.8) over the time interval $[0, T]$, and when

$$
\lim _{T \rightarrow T_{\mathrm{m}}}\left(\|(A, Q)\|_{\mathcal{E}(0, T)}+\max \left\{\left|A_{i}(x, T)\right|^{-1} \mid 1 \leqslant i \leqslant 3, x \in[0,1]\right\}\right)=\infty .
$$

We are now in a position to state the main result of the paper in the case of the simple network represented in Figure 1.

Theorem 1.2. Let us assume that, for $i=1,2,3, A_{i}^{0}>0, A^{0} \in\left[H^{1}(I)\right]^{3}, Q^{0} \in\left[H^{1}(I)\right]^{3}, h_{i} \in H_{\mathrm{loc}}^{3 / 4}([0, \infty))$, and that the following compatibility conditions are satisfied

$$
\begin{aligned}
& Q_{1}^{0}(1)=Q_{2}^{0}(0)+Q_{3}^{0}(0), \\
& Q_{1}^{0}(0)=h_{1}(0), \quad Q_{2}^{0}(1)=h_{2}(0), \quad Q_{3}^{0}(1)=h_{3}(0) .
\end{aligned}
$$

Then, the system (1.8) admits a unique maximal strong solution over $\left[0, T_{\mathrm{m}}\right)$, for some $T_{\mathrm{m}}>0$. Both the solution and the maximal time of existence $T_{\mathrm{m}}$ are unique.

Remark 1.3. In the above theorem, we state the existence of a unique maximal solution for the system (1.4)(1.6) with Dirichlet boundary conditions on the flow rate (1.7). Later on, we shall prove the existence of maximal unique solution when the Dirichlet boundary conditions are replaced by nonlinear Dirichlet boundary conditions (see Theorem 5.3) and nonlinear Robin boundary conditions (see Theorem 6.2). These nonlinear Robin boundary conditions, which approximate Dirichlet boundary conditions, allow us to prove an energy estimate satisfied by the corresponding solutions (see Proposition 6.3). In Theorem 5.3, the nonlinear Dirichlet boundary conditions are introduced to take into account boundary conditions on the velocity.

To study the system (1.8), in Section 2, we rewrite it in the form of a linear system in which the nonlinear terms are collected in source terms. The existence and regularity results for the associated nonhomogeneous linear system are obtained in Section 3. The nonlinear terms are estimated in Section 4 and Theorem 1.2 is proved in Section 5 with the Banach fixed point Theorem. In Section 6, by adapting results obtained in [10] for the quasilinear hyperbolic system corresponding to our model when $\nu=0$, we prove an energy identity satisfied by strong solutions of system (1.4)-(1.7). This energy identity is not sufficient to obtain a stability estimate because of the nonhomogeneous Dirichlet boundary conditions in (1.7). We are able to prove that if, in (1.7), we replace the classical nonhomogeneous Dirichlet boundary conditions by nonlinear Robin boundary conditions, the associated nonlinear system admits a unique maximal strong solution, and that this solution satisfies a stability estimate (see Theorem 6.2 and Proposition 6.3). We generalize the previous results to general networks in Section 7. 


\section{ReFormulation of THE NONLINEAR SYSTEM}

In order to prove the existence of solutions to system (1.8), we rewrite it in the form of a linearized system in which the nonlinear terms of system (1.8) are right hand side terms in the equations. For that, for an arbitrary $\bar{A} \in\left[H^{1}(I)\right]^{3}$ satisfying $\gamma_{\bar{A}}>0$, we introduce the coefficients

$$
L_{\bar{A}}^{i}=\frac{\rho}{\bar{A}_{i}}, \quad N \frac{i}{A}=\frac{\nu}{2 A_{i, 0} \sqrt{\overline{\bar{A}}_{i}}}, \quad i=1,2,3,
$$

and, for $i=1,2,3$, we define the nonlinear terms $F_{\bar{A}}^{i}$ and $G_{\bar{A}}^{i}$ by

$$
\begin{aligned}
& F_{\bar{A}}^{i}\left(A_{i}, Q_{i}\right)=-k_{f} \rho \frac{Q_{i}}{A_{i}^{2}}-\frac{2 \rho Q_{i}}{A_{i}^{2}} \frac{\partial Q_{i}}{\partial x}+\frac{\rho Q_{i}^{2}}{A_{i}^{3}} \frac{\partial A_{i}}{\partial x}-\rho\left(\frac{1}{A_{i}}-\frac{1}{\bar{A}_{i}}\right) \frac{\partial Q_{i}}{\partial t} \\
& +\frac{\partial}{\partial x}\left[\left(\frac{\nu}{2 A_{i, 0} \sqrt{A_{i}}}-\frac{\nu}{2 A_{i, 0} \sqrt{\bar{A}_{i}}}\right) \frac{\partial Q_{i}}{\partial x}\right]-\frac{\beta}{2 A_{i, 0} \sqrt{A_{i}}} \frac{\partial A_{i}}{\partial x}-\frac{1}{4 A_{i, 0} A_{i}^{3 / 2}} \frac{\partial A_{i}}{\partial x} \frac{\partial Q_{i}}{\partial x},
\end{aligned}
$$

and

$$
\begin{aligned}
& G_{\bar{A}}^{1}\left(A_{1}, Q_{1}\right)=\left.\left[\frac{\beta}{A_{1,0}}\left(\sqrt{A_{1}}-\sqrt{\bar{A}_{1}}\right)+\frac{\nu}{2 A_{1,0}} \frac{\partial Q_{1}}{\partial x}\left(\frac{1}{\sqrt{\bar{A}_{1}}}-\frac{1}{\sqrt{A_{1}}}\right)+\frac{1}{2} \rho \frac{Q_{1}^{2}}{A_{1}^{2}}\right]\right|_{x=1}, \\
& G_{\bar{A}}^{i}\left(A_{i}, Q_{i}\right)=\left.\left[\frac{\beta}{A_{i, 0}}\left(\sqrt{A_{i}}-\sqrt{\bar{A}_{i}}\right)+\frac{\nu}{2 A_{i, 0}} \frac{\partial Q_{i}}{\partial x}\left(\frac{1}{\sqrt{\bar{A}_{i}}}-\frac{1}{\sqrt{A_{i}}}\right)+\frac{1}{2} \rho \frac{Q_{i}^{2}}{A_{i}^{2}}\right]\right|_{x=0}, \quad i=2,3 .
\end{aligned}
$$

Moreover, the constant terms are defined by

$$
\begin{aligned}
& g_{\frac{1}{A}}=\left.\left[\frac{\beta}{A_{1,0}}\left(\sqrt{\bar{A}_{1}}-\sqrt{A_{1,0}}\right)\right]\right|_{x=1}, \\
& g_{\frac{i}{A}}=\left.\left[\frac{\beta}{A_{i, 0}}\left(\sqrt{\bar{A}_{i}}-\sqrt{A_{i, 0}}\right)\right]\right|_{x=0}, \quad i=2,3 .
\end{aligned}
$$

From now on, to simplify the presentation, we are going to choose $\left(t_{0}, t_{1}\right)=(0, T)$, but all the results can be adapted to the case when $\left(t_{0}, t_{1}\right) \neq(0, T)$. With the nonlinear terms introduced above, the system (1.8) can now be rewritten as:

$$
\left\{\begin{array}{l}
\text { For } i \in\{1,2,3\},\left(A_{i}, Q_{i}\right) \text { satisfies } \\
\frac{\partial A_{i}}{\partial t}+\frac{\partial Q_{i}}{\partial x}=0, \quad t \in(0, T), x \in I, \\
L_{A^{0}}^{i} \frac{\partial Q_{i}}{\partial t}-\frac{\partial}{\partial x}\left(N_{A^{0}}^{i} \frac{\partial Q_{i}}{\partial x}\right)=F_{A^{0}}^{i}\left(A_{i}, Q_{i}\right), \quad t \in(0, T), x \in I \\
Q_{1}(1, t)=Q_{2}(0, t)+Q_{3}(0, t), \quad t \in(0, T), \\
Q_{1}(0, t)=h_{1}(t), \quad Q_{2}(1, t)=h_{2}(t), \quad Q_{3}(1, t)=h_{3}(t), \quad t \in(0, T), \\
-N_{A^{0}}^{1} \frac{\partial Q_{1}}{\partial x}(1, t)+g_{A^{0}}^{1}+G_{A^{0}}^{1}\left(A_{1}, Q_{1}\right)=-N_{A^{0}}^{2} \frac{\partial Q_{2}}{\partial x}(0, t)+g_{A^{0}}^{2}+G_{A^{0}}^{2}\left(A_{2}, Q_{2}\right) \\
\quad=-N_{A^{0}}^{3} \frac{\partial Q_{3}}{\partial x}(0, t)+g_{A^{0}}^{3}+G_{A^{0}}^{3}\left(A_{3}, Q_{3}\right), \quad t \in(0, T), \\
A_{i}(x, 0)=A_{i}^{0}(x), \quad Q_{i}(x, 0)=Q_{i}^{0}(x), \quad x \in I
\end{array}\right.
$$

In the above system, we use the nonlinear terms and the coefficients corresponding to $\bar{A}=A^{0}$. But in Section 5.1 , we shall need to study system $(1.8)$ over $(0, \widehat{T})$, and next over $(\widehat{T}, \tau)$. This is why it is important to express the dependence of the nonlinear terms and the coefficients on $A^{0}$, or on $A(\widehat{T})$. 


\section{Study of A LineAR MODEL}

The proof of Theorem 1.2 relies on the Banach fixed point Theorem. The idea is to replace the nonlinear terms in (2.5) by given source terms $f_{i}$ and $g_{i}$. Throughout this section, we assume that $\bar{A} \in\left[H^{1}(I)\right]^{3}$ and $\gamma_{\bar{A}}>0\left(\gamma_{\bar{A}}\right.$ is defined in $\left.(1.11)\right)$. To study system (2.5), we are going to establish regularity results for the following linear system:

$$
\begin{cases}\text { For } i \in\{1,2,3\},\left(A_{i}, Q_{i}\right) \text { satisfies } & \\ \frac{\partial A_{i}}{\partial t}+\frac{\partial Q_{i}}{\partial x}=0 & t \in(0, T), x \in I \\ L_{\bar{i}}^{i} \frac{\partial Q_{i}}{\partial t}-\frac{\partial}{\partial x}\left(N \frac{i}{A} \frac{\partial Q_{i}}{\partial x}\right)=f_{i} & t \in(0, T), x \in I \\ Q_{1}(1, t)=Q_{2}(0, t)+Q_{3}(0, t) & t \in(0, T), \\ Q_{1}(0, t)=h_{1}(t), \quad Q_{2}(1, t)=h_{2}(t), \quad Q_{3}(1, t)=h_{3}(t), & t \in(0, T), \\ -N_{\overline{1}} \frac{\partial Q_{1}}{\partial x}(1, t)+g_{1}(t)=-N_{\frac{2}{A}} \frac{\partial Q_{2}}{\partial x}(0, t)+g_{2}(t)=-N_{\frac{3}{A}} \frac{\partial Q_{3}}{\partial x}(0, t)+g_{3}(t) & t \in(0, T), \\ A_{i}(x, 0)=A_{i}^{0}(x), \quad Q_{i}(x, 0)=Q_{i}^{0}(x) & x \in I\end{cases}
$$

Let us remark that the above linear system can be solved "in cascades": The equations (3.1) $3-6$ satisfied by $Q_{i}$, with the initial condition for $Q_{i}$ in $(3.1)_{7}$, can be solved independently of $A_{i}$. Once we have obtained the regularity of $Q_{i}$, we can easily get the regularity of $A_{i}$ from $(3.1)_{2}$. Thus, at first we concentrate in solving the following system with homogeneous boundary conditions:

$$
\begin{cases}\text { For } i \in\{1,2,3\}, Q_{i} \text { satisfies } & \\ L_{\bar{i}} \frac{\partial Q_{i}}{\partial t}-\frac{\partial}{\partial x}\left(N_{\bar{i}} \frac{\partial Q_{i}}{\partial x}\right)=f_{i} & t \in(0, T), x \in I, \\ Q_{1}(1, t)=Q_{2}(0, t)+Q_{3}(0, t) & t \in(0, T), \\ Q_{1}(0, t)=Q_{2}(1, t)=Q_{3}(1, t)=0 & t \in(0, T), \\ -N_{\frac{1}{A}} \frac{\partial Q_{1}}{\partial x}(1, t)=-N_{\frac{2}{A}} \frac{\partial Q_{2}}{\partial x}(0, t)=-N_{\frac{3}{A}} \frac{\partial Q_{3}}{\partial x}(0, t) & t \in(0, T), \\ Q_{i}(x, 0)=Q_{i}^{0}(x) & x \in I .\end{cases}
$$

3.1. Study of system (3.2). We first study the following stationary system:

We introduce the space

$$
\left\{\begin{array}{l}
\text { For } i \in\{1,2,3\}, Q_{i} \text { satisfies } \\
-\frac{\partial}{\partial x}\left(N \frac{i}{A} \frac{\partial Q_{i}}{\partial x}\right)=f_{i} \quad \text { in } I, \\
Q_{1}(1)=Q_{2}(0)+Q_{3}(0), \\
Q_{1}(0)=Q_{2}(1)=Q_{3}(1)=0, \\
-N_{\frac{1}{A}} \frac{\mathrm{d} Q_{1}}{\mathrm{~d} x}(1)=-N_{\frac{2}{A}} \frac{\mathrm{d} Q_{2}}{\mathrm{~d} x}(0)=-N_{\frac{3}{A}} \frac{\mathrm{d} Q_{3}}{\mathrm{~d} x}(0) .
\end{array}\right.
$$

$$
V=\left\{\left(Q_{1}, Q_{2}, Q_{3}\right) \in\left[H^{1}(I)\right]^{3} \mid Q_{1}(0)=0=Q_{2}(1)=Q_{3}(1), \quad Q_{1}(1)=Q_{2}(0)+Q_{3}(0)\right\},
$$

and the bilinear form ' $a$ ' defined on $V \times V$ by

$$
a(Q, \Phi)=\sum_{i=1}^{3} \int_{0}^{1} N \frac{i}{A} \frac{\mathrm{d} Q_{i}}{\mathrm{~d} x} \frac{\mathrm{d} \Phi_{i}}{\mathrm{~d} x}, \quad \Phi=\left(\Phi_{1}, \Phi_{2}, \Phi_{3}\right) .
$$

Lemma 3.1. A function $Q \in\left(H^{2}(I)\right)^{3}$ satisfies (3.3) if and only if it is solution of the following variational problem

$$
\text { Determine } Q \in V \quad \text { such that } \quad a(Q, \Phi)=\sum_{i=1}^{3} \int_{0}^{1} f_{i} \Phi_{i} \mathrm{~d} x \quad \text { for all } \Phi \in V \text {. }
$$


Proof. If $Q \in\left(H^{2}(I)\right)^{3}$ is solution of (3.3), then an integration by parts gives

$$
a(Q, \Phi)+\sum_{i=1}^{3}\left[-N \frac{i}{A} \frac{\mathrm{d} Q_{i}}{\mathrm{~d} x}(1) \Phi_{i}(1)+N \frac{i}{A} \frac{\mathrm{d} Q_{i}}{\mathrm{~d} x}(0) \Phi_{i}(0)\right]=\sum_{i=1}^{3} \int_{0}^{1} f_{i} \Phi_{i} \mathrm{~d} x
$$

Using the condition $\Phi_{1}(0)=0=\Phi_{2}(1)=\Phi_{3}(1)$ and the Neumann boundary conditions $(3.3)_{5}$ satisfied by $\left(Q_{1}, Q_{2}, Q_{3}\right)$, we have

$$
\begin{aligned}
& \sum_{i=1}^{3}\left[-N_{\frac{i}{A}} \frac{\mathrm{d} Q_{i}}{\mathrm{~d} x}(1) \Phi_{i}(1)+N \frac{i}{A} \frac{\mathrm{d} Q_{i}}{\mathrm{~d} x}(0) \Phi_{i}(0)\right] \\
& =-N_{\frac{2}{A}} \frac{\mathrm{d} Q_{2}}{\mathrm{~d} x}(0) \Phi_{1}(1)+N_{\frac{2}{A}} \frac{\mathrm{d} Q_{2}}{\mathrm{~d} x}(0) \Phi_{2}(0)+N_{\frac{3}{A}} \frac{\mathrm{d} Q_{3}}{\mathrm{~d} x}(0) \Phi_{3}(0) \\
& =\left[-N_{\frac{2}{A}} \frac{\mathrm{d} Q_{2}}{\mathrm{~d} x}(0)+N_{\frac{3}{A}} \frac{\mathrm{d} Q_{3}}{\mathrm{~d} x}(0)\right] \Phi_{3}(0)=0 .
\end{aligned}
$$

Thus, $Q$ is a solution to the variational problem (3.4).

The converse statement can be proved in a classical way by first recovering equation $(3.3)_{2}$, and next the regularity of $\left(Q_{i}\right)_{i=1}^{3}$ and the boundary conditions $(3.3)_{3-5}$. The proof is complete.

Proposition 3.2. Let us assume that, for $i=1,2,3, f_{i} \in L^{2}(I)$. Then the system (3.3) admits a unique solution $Q \in\left(H^{2}(I)\right)^{3}$ and

$$
\sum_{i=1}^{3}\left\|Q_{i}\right\|_{H^{2}(I)} \leqslant C \sum_{i=1}^{3}\left\|f_{i}\right\|_{L^{2}(I)}
$$

Proof. The bilinear form $a$ is continuous and coercive on $V \times V$. The existence of a unique weak solution to the variational problem (3.4) follows from the Lax-Milgram Lemma. The end of proof is classical.

We introduce the unbounded operator $(\mathcal{A}, \mathcal{D}(\mathcal{A}))$ in $\left[L^{2}(I)\right]^{3}$ defined by

$$
\begin{aligned}
& \mathcal{D}(\mathcal{A})=\left\{Q \in V \cap\left[H^{2}(I)\right]^{3} \mid Q=\left(Q_{1}, Q_{2}, Q_{3}\right) \text { satisfies }(3.3)_{5}\right\}, \\
& \mathcal{A} Q=\left(\mathcal{A}_{1} Q_{1}, \mathcal{A}_{2} Q_{2}, \mathcal{A}_{3} Q_{3}\right) \quad \text { where } \\
& \mathcal{A}_{i} Q_{i}=-\frac{\partial}{\partial x}\left(N_{i} \frac{\partial Q_{i}}{\partial x}\right) \quad \text { for } i=1,2,3 .
\end{aligned}
$$

The following proposition is an easy consequence of Proposition 3.2 and of its proof.

Proposition 3.3. The operator $(\mathcal{A}, \mathcal{D}(\mathcal{A}))$ is the infinitesimal generator of a strongly continuous analytic semigroup on $\left[L^{2}(I)\right]^{3}$. Moreover, $(\mathcal{A}, \mathcal{D}(\mathcal{A}))$ is a self-adjoint operator with compact resolvent in $\left[L^{2}(I)\right]^{3}$.

To define weak solutions to system (3.2), we introduce the following spaces

$$
\begin{aligned}
H(0, T) & =\left[H^{1}\left(0, T ; L^{2}(I)\right)\right]^{3}, \\
W(0, T) & =\left\{Q \in L^{2}(0, T ; V) \mid \frac{\mathrm{d} Q}{\mathrm{~d} t} \in L^{2}\left(0, T ; V^{\prime}\right)\right\},
\end{aligned}
$$

where $V^{\prime}$ is the dual of $V$ with respect to the pivot space $\left(L^{2}(I)\right)^{3}$.

Definition 3.4. We say that $Q \in W(0, T)$ is a weak solution to (3.2) if the following conditions hold

$$
\begin{aligned}
& \sum_{i=1}^{3}\left(\left\langle L_{\frac{i}{A}} \frac{\mathrm{d} Q_{i}}{\mathrm{~d} t}(t), \xi_{i}\right\rangle_{V^{\prime}, V}+\int_{0}^{1} N_{\frac{i}{A}} \frac{\partial Q_{i}}{\partial x} \frac{\partial \xi_{i}}{\partial x} \mathrm{~d} x\right)=\sum_{i=1}^{3} \int_{0}^{1} f_{i} \xi_{i} \mathrm{~d} x \text { for all } \xi=\left(\xi_{i}\right)_{i=1}^{3} \in V \\
& Q_{i}(\cdot, 0)=Q_{i}^{0} .
\end{aligned}
$$

The main result of this subsection is the following: 
Theorem 3.5. Let $f_{i} \in L^{2}\left(0, T ; L^{2}(I)\right)$ and $Q^{0} \in V$. Then there exists a unique weak solution $Q \in W(0, T)$ to (3.2). Moreover, we have

$$
Q_{i} \in L^{2}\left(0, T ; H^{2}(I)\right) \cap H^{1}\left(0, T ; L^{2}(I)\right) \cap L^{\infty}\left(0, T ; H^{1}(I)\right),
$$

and

$$
\begin{aligned}
\sum_{i=1}^{3}\left[\left\|Q_{i}\right\|_{L^{\infty}\left(0, T ; H^{1}(I)\right)}+\left\|Q_{i}\right\|_{L^{2}\left(0, T ; H^{2}(I)\right)}+\left\|Q_{i}\right\|_{H^{1}\left(0, T ; L^{2}(I)\right)}\right] & \\
& \leqslant C \sum_{i=1}^{3}\left(\left\|f_{i}\right\|_{L^{2}\left(0, T ; L^{2}(I)\right)}+\left\|Q_{i}^{0}\right\|_{H^{1}(I)}\right),
\end{aligned}
$$

where the constant $C$ depends on $L_{i}, N_{i}$, but is independent of $T$.

Proof. Due to Proposition 3.3, there exists a Hilbertian basis in $\left(L^{2}(I)\right)^{3}$, namely $\left(\xi_{1, k}, \xi_{2, k}, \xi_{3, k}\right)_{k \in \mathbb{N}^{*}}$, constituted of eigenfunctions of $\mathcal{A}$, such that $\left(\xi_{1, k}, \xi_{2, k}, \xi_{3, k}\right)$ belongs to $\mathcal{D}(\mathcal{A})$ for all $k \in \mathbb{N}^{*}$. Using that basis, we can follow the lines of the proof of [8, Theorem 4, Chapter 7] to prove the existence of a unique weak solution to system $(3.2)$ in $W(0, T)$.

The estimate in (3.9) can be proved as in [8, Theorem 5, Chapter 7].

3.2. Study of system (3.1). We want to study the regularity of solutions of the linear problem (3.1).

Lemma 3.6. Let us assume that, for $i=1,2,3, h_{i} \in H_{\mathrm{loc}}^{3 / 4}([0, \infty)), g_{i} \in H_{\mathrm{loc}}^{1 / 4}([0, \infty)), Q_{i}^{0} \in H^{1}(I)$, and the compatibility conditions (1.16) are satisfied.

Then, for $i=1,2,3$, there exists a function $Q_{i} \in L_{\mathrm{loc}}^{2}\left([0, \infty) ; H^{2}(I)\right) \cap H_{\mathrm{loc}}^{1}\left([0, \infty) ; L^{2}(I)\right)$ $\cap L_{\mathrm{loc}}^{\infty}\left([0, \infty) ; H^{1}(I)\right)$ such that

$$
\begin{array}{lll}
Q_{1}(0, t)=h_{1}(t), \quad Q_{1}(1, t)=0, & \frac{\partial Q_{1}}{\partial x}(1, t)=g_{1}(t), \quad Q_{1}(\cdot, 0)=Q_{1}^{0}, \\
Q_{2}(0, t)=0, \quad Q_{2}(1, t)=h_{2}(t), & \frac{\partial Q_{2}}{\partial x}(0, t)=g_{2}(t), \quad Q_{2}(\cdot, 0)=Q_{2}^{0}, \\
Q_{3}(0, t)=0, \quad Q_{3}(1, t)=h_{3}(t), & \frac{\partial Q_{3}}{\partial x}(0, t)=g_{3}(t), \quad Q_{3}(\cdot, 0)=Q_{3}^{0}, \quad \text { for all } t \geqslant 0 .
\end{array}
$$

Moreover, there exists a constant $C>0$, independent of $T>0$, such that, for $i=1,2,3$, we have

$$
\left\|Q_{i}\right\|_{L^{2}\left(0, T ; H^{2}(I)\right) \cap H^{1}\left(0, T ; L^{2}(I)\right) \cap L^{\infty}\left(0, T ; H^{1}(I)\right)} \leqslant C\left(\left\|g_{i}\right\|_{H^{1 / 4}(0, T)}+\left\|h_{i}\right\|_{H^{3 / 4}(0, T)}+\left\|Q_{i}^{0}\right\|_{H^{1}(I)}\right) .
$$

Proof. The proof of the above lemma follows from [15, Theorem 2.3, page 18].

We prove the following theorem:

Theorem 3.7. Let us assume that, for $i=1,2,3, h_{i} \in H_{\mathrm{loc}}^{3 / 4}([0, \infty))$ and $g_{i} \in H_{\mathrm{loc}}^{1 / 4}([0, \infty)), f_{i} \in L^{2}\left(0, T ; L^{2}(I)\right)$, and the following compatibility conditions are satisfied

$$
Q_{1}^{0}(1)=Q_{2}^{0}(0)+Q_{3}^{0}(0), \quad Q_{1}^{0}(0)=h_{1}(0), \quad Q_{2}^{0}(1)=h_{2}(0), \quad Q_{3}^{0}(1)=h_{3}(0) .
$$

Then, the system (3.1) admits a unique solution $(A, Q)$ satisfying

$$
\begin{aligned}
& \frac{\partial A_{i}}{\partial t} \in L^{\infty}\left(0, T ; L^{2}(I)\right), \quad A_{i} \in H^{1}\left(0, T ; H^{1}(I)\right), \\
& Q_{i} \in L^{2}\left(0, T ; H^{2}(I)\right) \cap H^{1}\left(0, T ; L^{2}(I)\right) \cap L^{\infty}\left(0, T ; H^{1}(I)\right), \quad i=1,2,3 .
\end{aligned}
$$


Furthermore, there exists a constant $C_{\bar{A}}$, depending on $\bar{A}$ but independent of $T$, such that

$$
\begin{gathered}
\sum_{i=1}^{3}\left[\left\|\frac{\partial A_{i}}{\partial t}\right\|_{L^{\infty}\left(0, T ; L^{2}(I)\right)}+\left\|A_{i}\right\|_{H^{1}\left(0, T ; H^{1}(I)\right)}+\left\|A_{i}\right\|_{L^{\infty}\left(0, T ; H^{1}(I)\right)}\right. \\
\left.+\left\|Q_{i}\right\|_{L^{\infty}\left(0, T ; H^{1}(I)\right)}+\left\|Q_{i}\right\|_{L^{2}\left(0, T ; H^{2}(I)\right)}+\left\|Q_{i}\right\|_{H^{1}\left(0, T ; L^{2}(I)\right)}\right] \\
\leqslant C_{\bar{A}} \sum_{i=1}^{3}\left(\left\|f_{i}\right\|_{L^{2}\left(0, T ; L^{2}(I)\right)}+\left\|g_{i}\right\|_{H^{1 / 4}(0, T)}+\left\|h_{i}\right\|_{H^{3 / 4}(0, T)}+\left\|A_{i}^{0}\right\|_{H^{1}(I)}+\left\|Q_{i}^{0}\right\|_{H^{1}(I)}\right)
\end{gathered}
$$

for all $T>0$.

Proof. We first study the existence and uniqueness of a solution $\left(Q_{1}, Q_{2}, Q_{3}\right)$ in $\left[L^{2}\left(0, T ; H^{2}(I)\right)\right]^{3} \cap$ $\left[H^{1}\left(0, T ; L^{2}(I)\right)\right]^{3} \cap\left[L^{\infty}\left(0, T ; H^{1}(I)\right]^{3}\right.$ to system $(3.1)_{3-7}$. Let $\left(\widetilde{Q}_{1}, \widetilde{Q}_{2}, \widetilde{Q}_{3}\right)$ be a solution to (3.10) satisfying (3.11), i.e,

$$
\left\|\widetilde{Q}_{i}\right\|_{L^{2}\left(0, T ; H^{2}(I)\right) \cap H^{1}\left(0, T ; L^{2}(I)\right) \cap L^{\infty}\left(0, T ; H^{1}(I)\right)} \leqslant C\left(\left\|g_{i}\right\|_{H^{1 / 4}(0, T)}+\left\|h_{i}\right\|_{H^{3 / 4}(0, T)}+\left\|Q_{i}^{0}\right\|_{H^{1}(I)}\right) .
$$

We look for $Q=\left(Q_{i}\right)_{i=1}^{3}$ in the form $\left(Q_{1}, Q_{2}, Q_{3}\right)=\left(\widetilde{Q}_{1}, \widetilde{Q}_{2}, \widetilde{Q}_{3}\right)+\left(\widehat{Q}_{1}, \widehat{Q}_{2}, \widehat{Q}_{3}\right)$. Thus, $\widehat{Q}=\left(\widehat{Q}_{1}, \widehat{Q}_{2}, \widehat{Q}_{3}\right)$ satisfies

$$
\begin{cases}L_{\frac{i}{A}} \frac{\partial \widehat{Q}_{i}}{\partial t}-\frac{\partial}{\partial x}\left(N \frac{i}{A} \frac{\partial \widehat{Q}_{i}}{\partial x}\right)=\widehat{f}_{i} & t \in(0, T), x \in I, i \in\{1,2,3\}, \\ \widehat{Q}_{1}(1, t)=\widehat{Q}_{2}(0, t)+\widehat{Q}_{3}(0, t) & t \in(0, T), \\ \widehat{Q}_{1}(0, t)=\widehat{Q}_{2}(1, t)=\widehat{Q}_{3}(1, t)=0 & t \in(0, T), \\ -N_{\frac{1}{A}} \frac{\partial \widehat{Q}_{1}}{\partial x}(1, t)=-N_{\frac{2}{A}} \frac{\partial \widehat{Q}_{2}}{\partial x}(0, t)=-N_{\frac{3}{A}} \frac{\partial \widehat{Q}_{3}}{\partial x}(0, t) & t \in(0, T), \\ \widehat{Q}_{i}(x, 0)=0 & x \in I, i \in\{1,2,3\},\end{cases}
$$

where

$$
\widehat{f}_{i}=f_{i}-L_{\bar{A}}^{i} \frac{\partial \widetilde{Q}_{i}}{\partial t}+\frac{\partial}{\partial x}\left(N_{\bar{i}}^{i} \frac{\partial \widetilde{Q}_{i}}{\partial x}\right)
$$

With Theorem 3.5, we have

$$
\sum_{i=1}^{3}\left[\left\|\widehat{Q}_{i}\right\|_{L^{\infty}\left(0, T ; H^{1}(I)\right)}+\left\|\widehat{Q}_{i}\right\|_{L^{2}\left(0, T ; H^{2}(I)\right)}+\left\|\widehat{Q}_{i}\right\|_{H^{1}\left(0, T ; L^{2}(I)\right)}\right] \leqslant C \sum_{i=1}^{3}\left\|\widehat{f}_{i}\right\|_{L^{2}\left(0, T ; L^{2}(I)\right)},
$$

The above estimate together with (3.15) give

$$
\begin{aligned}
& \sum_{i=1}^{3}\left[\left\|Q_{i}\right\|_{L^{\infty}\left(0, T ; H^{1}(I)\right)}+\left\|Q_{i}\right\|_{L^{2}\left(0, T ; H^{2}(I)\right)}+\left\|Q_{i}\right\|_{H^{1}\left(0, T ; L^{2}(I)\right)}\right] \\
& \quad \leqslant C \sum_{i=1}^{3}\left(\left\|f_{i}\right\|_{L^{2}\left(0, T ; L^{2}(I)\right)}+\left\|g_{i}\right\|_{H^{1 / 4}(0, T)}+\left\|h_{i}\right\|_{H^{3 / 4}(0, T)}+\left\|Q_{i}^{0}\right\|_{H^{1}(I)}\right)
\end{aligned}
$$

The estimate for $\left(A_{i}\right)_{i=1}^{3}$ is obtained with the help of the first equation of system (3.1).

\section{Estimates OF NONLINEAR TERMS}

Throughout this section, for $0<T \leqslant \infty$, we set

$$
I_{T}=I \times(0, T), \quad H^{2,1}\left(I_{T}\right)=L^{2}\left(0, T ; H^{2}(I)\right) \cap H^{1}\left(0, T ; L^{2}(I)\right) .
$$


4.1. Preliminary results. Let us recall some important lemmas which will be used later on.

Lemma 4.1. Let $1 / 2<s \leqslant 1$. There is a bounded extension operator from $\left\{f \in H^{s}(0, T) \mid\right.$ $f(0)=0\}$ to $H^{s}(0, \infty)$, uniformly bounded with respect to $T>0$.

Lemma 4.2. (i) Let us assume that $g \in H^{s_{2}}(0, T)$ with $g(0)=0$ and $s_{2}>s_{1}, s_{1}, s_{2} \in(1 / 2,1]$. Then there exists a constant $C>0$ independent of $T$ such that

$$
\|g\|_{H^{s_{1}(0, T)}} \leqslant C(\sqrt{T})^{1-s_{1} / s_{2}}\|g\|_{H^{s_{2}(0, T)}} .
$$

(ii) Let us assume that $f \in H^{s_{1}}(0, T), g \in H^{s_{2}}(0, T)$ with $s_{1}, s_{2} \in(1 / 2,1]$ and $f(0)=g(0)=0$. Then there exists a constant $C>0$, independent of $T$ such that $f g \in H^{s}(0, T)$ for $s \in(1 / 2,1)$ with $s_{1}+s_{2}-s>1 / 2$ and

$$
\|f g\|_{H^{s}(0, T)} \leqslant C T^{\delta}\|f\|_{H^{s_{1}(0, T)}}\|g\|_{H^{s_{2}(0, T)}}
$$

for some $\delta>0$.

(iii) Let us assume that $f \in H^{s_{1}}(0, T), g \in H^{s_{2}}(0, T)$ with $s_{1}, s_{2} \in(1 / 2,1]$ and $g(0)=0$. Then there exists a constant $C>0$, independent of $T$ such that $f g \in H^{s}\left(0, T ; U_{3}\right)$ for $s \in(1 / 2,1)$ with $s_{1}+s_{2}-s>1 / 2$ and

$$
\|f g\|_{H^{s}(0, T)} \leqslant C T^{\delta}\left(\|f\|_{H^{s_{1}(0, T)}}+|f(0)|+\|g\|_{H^{s_{2}(0, T)}}\right)
$$

for some $\delta>0$.

Proof. Step 1. Let us first prove (4.1). Let $\bar{g}$ be the extension of $g$ to $(0, \infty)$ introduced in Lemma 4.1. Since $g(0)=0$, we have from Lemma 4.1 that

$$
\|g\|_{L^{2}(0, T)} \leqslant \sqrt{T}\|g\|_{L^{\infty}(0, T)} \leqslant \sqrt{T}\|\bar{g}\|_{L^{\infty}(0, \infty)} \leqslant \sqrt{T}\|\bar{g}\|_{H^{s_{2}(0, \infty)}} \leqslant C \sqrt{T}\|g\|_{H^{s_{2}(0, T)}} .
$$

Let us now fix $1 / 2<s_{1}<1$. By interpolation and the above inequality, we have

$$
\|g\|_{H^{s_{1}(0, T)}} \leqslant\|\bar{g}\|_{H^{s_{1}(0, \infty)}} \leqslant\|\bar{g}\|_{L^{2}(0, \infty)}^{1-s_{1} / s_{2}}\|\bar{g}\|_{H^{s_{2}(0, \infty)}}^{s_{1} / s_{2}} \leqslant C\|g\|_{L^{2}(0, T)}^{1-s_{1} / s_{2}}\|g\|_{H^{s_{2}(0, T)}}^{s_{1} / s_{2}}
$$

$$
\leqslant C(\sqrt{T})^{1-s_{1} / s_{2}}\|g\|_{H^{s_{2}(0, T)}}
$$

with $C$ independent of $T$ since $g(0)=0$.

Step 2. We want to prove (4.2). We have $\bar{f}, \bar{g}$ as extensions of $f, g$ to $(0, \infty)$ respectively following Lemma 4.1. As $s_{1}+s_{2}-s>1 / 2$, there exists $\varepsilon>0$ such that $s_{1}+s_{2}-s-\varepsilon>1 / 2$.

With [12, Proposition B1] and the estimate (4.1), we have

$$
\begin{aligned}
\|f g\|_{H^{s}(0, T)} \leqslant\|\bar{f} \bar{g}\|_{H^{s}(0, \infty)} \leqslant C\|\bar{f}\|_{H^{s_{1}(0, \infty)}}\|\bar{g}\|_{H^{s_{2}-\varepsilon}(0, \infty)} & \leqslant C\left|\|f\|_{H^{s_{1}(0, T)}}\right|\|g\|_{H^{s_{2}-\varepsilon}(0, T)} \leqslant T^{\delta}\left|\|f\|_{H^{s_{1}(0, T)}}\right|\|g\|_{H^{s_{2}(0, T)}}, \\
& \leqslant(0)
\end{aligned}
$$

where the constant $C$ is independent of $T$ as $f(0)=g(0)=0$.

Step 3. To prove (4.3), we first write $f g$ as

$$
f g=(f-f(0)) g+f(0) g \text {. }
$$

Now we can use (4.2) to prove (4.3).

Lemma 4.3. There exists a constant $C>0$, independent of $T>0$ and $0<\gamma \leqslant 1$, such that, for all $f \in H^{1}\left(0, T ; H^{1}(I)\right)$ and $f(x, t) \geqslant \gamma$ in $I \times(0, T)$, the functions $\sqrt{f}, f^{1 / 4}$ and $1 / f$ belong to $H^{1}\left(0, T ; H^{1}(I)\right)$ and the following estimates hold:

$$
\begin{aligned}
\|\sqrt{f}\|_{H^{1}\left(0, T ; H^{1}(I)\right)}+\|\sqrt{f}\|_{L^{\infty}\left(0, T ; H^{1}(I)\right)} \leqslant \frac{C}{\gamma^{3 / 2}}\left(\|f\|_{H^{1}\left(0, T ; H^{1}(I)\right)}\right. & +\|f\|_{L^{\infty}\left(0, T ; H^{1}(I)\right)} \\
& \left.+\|f\|_{H^{1}\left(0, T ; H^{1}(I)\right)}\|f\|_{L^{\infty}\left(0, T ; H^{1}(I)\right)}\right), \\
\left\|f^{1 / 4}\right\|_{H^{1}\left(0, T ; H^{1}(I)\right)}+\left\|f^{1 / 4}\right\|_{L^{\infty}\left(0, T ; H^{1}(I)\right)} \leqslant \frac{C}{\gamma^{7 / 4}}\left(\|f\|_{H^{1}\left(0, T ; H^{1}(I)\right)}\right. & +\|f\|_{L^{\infty}\left(0, T ; H^{1}(I)\right)} \\
& \left.+\|f\|_{H^{1}\left(0, T ; H^{1}(I)\right)}\|f\|_{L^{\infty}\left(0, T ; H^{1}(I)\right)}\right),
\end{aligned}
$$


and

$$
\begin{aligned}
\|1 / f\|_{H^{1}\left(0, T ; H^{1}(I)\right)}+\|1 / f\|_{L^{\infty}\left(0, T ; H^{1}(I)\right)} \leqslant \frac{C}{\gamma^{3}}\left(\|f\|_{H^{1}\left(0, T ; H^{1}(I)\right)}\right. & +\|f\|_{L^{\infty}\left(0, T ; H^{1}(I)\right)} \\
& \left.+\|f\|_{H^{1}\left(0, T ; H^{1}(I)\right)}\|f\|_{L^{\infty}\left(0, T ; H^{1}(I)\right)}\right) .
\end{aligned}
$$

Proof. The proof is easy and left to the reader.

Lemma 4.4. There exists a constant $C>0$ such that, for all $T>0$ and all $f$ belonging to $H^{2,1}\left(I_{T}\right)$, the following estimates hold

$$
\begin{aligned}
& \left\|\frac{\partial f}{\partial x}(0, \cdot)\right\|_{H^{1 / 4}(0, T)}+\left\|\frac{\partial f}{\partial x}(1, \cdot)\right\|_{H^{1 / 4}(0, T)} \leqslant C\left(\|f(\cdot, 0)\|_{H^{1}(I)}+\|f\|_{H^{2,1}\left(I_{T}\right)}\right), \\
& \|f(0, \cdot)\|_{H^{3 / 4}(0, T)}+\|f(1, \cdot)\|_{H^{3 / 4}(0, T)} \leqslant C\left(\|f(\cdot, 0)\|_{H^{1}(I)}+\|f\|_{H^{2,1}\left(I_{T}\right)}\right) .
\end{aligned}
$$

Proof. If $f \in H^{2,1}\left(I_{T}\right)$, then the fact that $\frac{\partial f}{\partial x}(0, \cdot)$ and $\frac{\partial f}{\partial x}(1, \cdot)$ belong to $H^{1 / 4}(0, T)$ can be deduced from [13, Chapter II, Lemma 3.4] or [11, Theorem 6.1], and the following estimate holds

$$
\left\|\frac{\partial f}{\partial x}(0, \cdot)\right\|_{H^{1 / 4}(0, T)}+\left\|\frac{\partial f}{\partial x}(1, \cdot)\right\|_{H^{1 / 4}(0, T)} \leqslant C_{T}\left(\|f\|_{L^{2}\left(0, T ; H^{2}(I)\right) \cap H^{1}\left(0, T ; L^{2}(I)\right.}\right),
$$

with a constant $C_{T}$ which may depend on $T$. To obtain a constant independent of $T$, we proceed as follows. With [13, Chapter II, Lemma 3.4]), we know that $f(\cdot, 0)$ belongs to $H^{1}(I)$. For some large $\lambda>0$, we consider the following problem

$$
\frac{\partial u}{\partial t}-\frac{\partial^{2} u}{\partial x^{2}}+\lambda u=0 \text { in } I_{\infty}, \quad \frac{\partial u}{\partial x}(0, t)=\frac{\partial u}{\partial x}(1, t)=0 \text { for all } t \geqslant 0, \quad u(x, 0)=f(x, 0) \text { for all } x \in I .
$$

Due to [2, Part II, Chapter 1, Theorem 3.1], $u$ belongs to $H^{2,1}\left(I_{\infty}\right)$ and

$$
\|u\|_{H^{2,1}\left(I_{T}\right)} \leqslant\|u\|_{H^{2,1}\left(I_{\infty}\right)} \leqslant C\|f(\cdot, 0)\|_{H^{1}(I)},
$$

with $I_{T}=I \times(0, T)$. Let us set $\widetilde{u}=u-f$. Then $\widetilde{u} \in H^{2,1}\left(I_{T}\right)$ and $\widetilde{u}(x, 0)=0$ for all $x \in I$. Then, due to Lemma 4.1 , there exists $u^{*} \in H^{2,1}\left(I_{\infty}\right)$ such that $u^{*}=\widetilde{u}$ in $[0, T]$ and

$$
\left\|u^{*}\right\|_{H^{2,1}\left(I_{\infty}\right)} \leqslant C\|\widetilde{u}\|_{H^{2,1}\left(I_{T}\right)} .
$$

Moreover, using [11, Theorem 6.1], we obtain

$$
\begin{aligned}
& \left\|\frac{\partial \widetilde{u}}{\partial x}(0, \cdot)\right\|_{H^{1 / 4}(0, T)}+\left\|\frac{\partial \widetilde{u}}{\partial x}(1, \cdot)\right\|_{H^{1 / 4}(0, T)}=\left\|\frac{\partial u^{*}}{\partial x}(0, \cdot)\right\|_{H^{1 / 4}(0, T)}+\left\|\frac{\partial u^{*}}{\partial x}(1, \cdot)\right\|_{H^{1 / 4}(0, T)} \\
& \leqslant\left\|\frac{\partial u^{*}}{\partial x}(0, \cdot)\right\|_{H^{1 / 4}(0, \infty)}+\left\|\frac{\partial u^{*}}{\partial x}(1, \cdot)\right\|_{H^{1 / 4}(0, \infty)} \leqslant C\left\|u^{*}\right\|_{H^{2,1}\left(I_{\infty}\right)} \leqslant C\|\widetilde{u}\|_{H^{2,1}\left(I_{T}\right)},
\end{aligned}
$$

where the constant $C$ is independent of $T$. Finally, the above estimate and (4.9) yield

$$
\begin{aligned}
& \left\|\frac{\partial f}{\partial x}(0, \cdot)\right\|_{H^{1 / 4}(0, T)}+\left\|\frac{\partial f}{\partial x}(1, \cdot)\right\|_{H^{1 / 4}(0, T)}=\left\|\frac{\partial \widetilde{u}}{\partial x}(0, \cdot)\right\|_{H^{1 / 4}(0, T)}+\left\|\frac{\partial \widetilde{u}}{\partial x}(1, \cdot)\right\|_{H^{1 / 4}(0, T)} \\
& \leqslant C\|\widetilde{u}\|_{H^{2,1}\left(I_{T}\right)} \leqslant C\left(\|u\|_{H^{2,1}\left(I_{T}\right)}+\|f\|_{H^{2,1}\left(I_{T}\right)}\right) \leqslant C\left(\|f(\cdot, 0)\|_{H^{1}(I)}+\|f\|_{H^{2,1}\left(I_{T}\right)}\right) .
\end{aligned}
$$

This completes the proof of estimate (4.7). Estimate (4.8) can be obtained in a similar manner. 


\subsection{Analysis of nonlinear terms. Let}

$$
\bar{A} \in\left[H^{1}(I)\right]^{3} \quad \text { such that } \gamma_{\bar{A}}>0,
$$

where $\gamma_{\bar{A}}$ is defined in (1.11). For $0 \leqslant t_{0}<t_{1}$ and $\gamma>0$, we introduce the space

$$
B_{\bar{A}}\left(t_{0}, t_{1} ; \gamma\right)=\left\{(A, Q) \in \mathcal{E}\left(t_{0}, t_{1}\right) \mid A \in E_{\bar{A}}\left(t_{0}, t_{1} ; \gamma\right)\right\},
$$

where $E_{\bar{A}}\left(t_{0}, t_{1} ; \gamma\right)$ is defined in (1.14). The ball $B_{\bar{A}}\left(t_{0}, t_{1} ; \gamma, \mu\right)$ in $B_{\bar{A}}\left(t_{0}, t_{1} ; \gamma\right)$ is defined as follows

$$
B_{\bar{A}}\left(t_{0}, t_{1} ; \gamma, \mu\right)=\left\{(A, Q) \in B_{\bar{A}}\left(t_{0}, t_{1} ; \gamma\right) \mid\|(A, Q)\|_{\mathcal{E}\left(t_{0}, t_{1}\right)} \leqslant \mu\right\} .
$$

Our aim is to obtain different estimates for the nonlinear terms $F_{\frac{i}{A}}, G_{\frac{i}{A}}$ introduced in $(2.2)-(2.4)$, in $I \times\left(t_{0}, t_{1}\right)$, for an arbitrary time interval $\left(t_{0}, t_{1}\right)$. For simplicity, we only treat the case when $\left(t_{0}, t_{1}\right)=(0, T), \gamma \in(0,1)$, and $\mu \geqslant 1$, but the results can be stated with obvious modifications for an arbitrary time interval $\left(t_{0}, t_{1}\right)$.

Now we prove the following proposition which will be used later on.

Proposition 4.5. There exists a constant $C>0$, independent of $\gamma \in(0,1)$, of $\mu \geqslant 1$, and of $T \in(0,1)$, such that, for all $(A, Q) \in B_{\bar{A}}(0, T ; \gamma, \mu)$, we have

$$
\begin{aligned}
& \left\|A_{i}-\bar{A}_{i}\right\|_{L^{\infty}\left(0, T ; H^{1}(I)\right)}+\left\|A_{i}\right\|_{L^{2}\left(0, T ; H^{1}(I)\right)} \leqslant C \mu \sqrt{T} \\
& \left\|\sqrt{A_{i}}-\sqrt{\bar{A}_{i}}\right\|_{L^{\infty}\left(0, T ; H^{1}(I)\right)}+\left\|\sqrt{A_{i}}\right\|_{L^{2}\left(0, T ; H^{1}(I)\right)} \leqslant C \mu \frac{\sqrt{T}}{\sqrt{\gamma}}, \\
& \left\|Q_{i}\right\|_{L^{2}\left(0, T ; H^{1+s}(I)\right)} \leqslant C \mu T^{(1-s) / 4}, \quad \text { for } s \in(0,1), \\
& \left\|Q_{i}\right\|_{L^{\infty}\left(I_{T}\right)}+\left\|A_{i}\right\|_{L^{\infty}\left(I_{T}\right)}+\left\|\sqrt{A_{i}}\right\|_{L^{\infty}\left(I_{T}\right)} \leqslant C \mu, \\
& \left\|\sqrt{A_{i}}-\sqrt{\bar{A}_{i}}\right\|_{H^{1}\left(0, T ; H^{1}(I)\right)} \leqslant C \mu \frac{\sqrt{T}}{\sqrt{\gamma}}, \\
& \left\|\left(A_{i}\right)^{2}-\left(\bar{A}_{i}\right)^{2}\right\|_{H^{1}\left(0, T ; H^{1}(I)\right)} \leqslant C \mu^{2} .
\end{aligned}
$$

Proof. We have

$$
A_{i}(x, t)-\bar{A}_{i}=\int_{0}^{t} \partial_{t} A_{i}(x, s) \mathrm{d} s \quad \text { and } \quad \sqrt{A_{i}}(x, t)-\sqrt{\bar{A}_{i}}=\int_{0}^{t} \partial_{t} \sqrt{A_{i}}(x, s) \mathrm{d} s .
$$

Therefore

$$
\left\|A_{i}-\bar{A}_{i}\right\|_{L^{\infty}\left(0, T ; H^{1}(0,1)\right)} \leqslant \sqrt{T}\left\|\partial_{t} A_{i}\right\|_{L^{2}\left(0, T ; H^{1}(0,1)\right)} \leqslant \mu \sqrt{T} .
$$

In a similar manner, we can easily obtain

$$
\left\|\sqrt{A_{i}}-\sqrt{\bar{A}_{i}}\right\|_{L^{\infty}\left(0, T ; H^{1}(0,1)\right)} \leqslant C \mu \frac{\sqrt{T}}{\sqrt{\gamma}} .
$$

Using (4.12), we first obtain

$$
\left\|A_{i}\right\|_{L^{\infty}\left(0, T ; H^{1}(I)\right)} \leqslant\left\|\bar{A}_{i}\right\|_{H^{1}(I)}+\left\|A_{i}-\bar{A}_{i}\right\|_{L^{\infty}\left(0, T ; H^{1}(I)\right)} \leqslant \mu+C \mu \sqrt{T},
$$

where the constant $C$ is independent of $T$. Thus

$$
\left\|A_{i}\right\|_{L^{2}\left(0, T ; H^{1}(0,1)\right)} \leqslant \sqrt{T}\left\|A_{i}\right\|_{L^{\infty}\left(0, T ; H^{1}(I)\right)} \leqslant C \mu(T+\sqrt{T}) .
$$

Similarly, we can show that $\left\|\sqrt{A_{i}}\right\|_{L^{2}\left(0, T ; H^{1}(0,1)\right)} \leqslant \mu+C \mu \frac{T}{\sqrt{\gamma}}$. The estimate (4.13) can be proved by following the arguments of [16, Proposition 6.4].

Estimate of $F_{\bar{A}}$. 
Proposition 4.6. For all $\mu \geqslant 1$ and $\gamma \in(0,1)$, there exists a positive constant $C_{F}(\mu, \gamma)$, depending on $\mu$ and $\gamma$, but independent of $T \in(0,1)$, such that, for all $(A, Q) \in B_{\bar{A}}(0, T ; \gamma, \mu)$, all $\left(A^{1}, Q^{1}\right) \in B_{\bar{A}}(0, T ; \gamma, \mu)$, and all $\left(A^{2}, Q^{2}\right) \in B_{\bar{A}}(0, T ; \gamma, \mu)$, we have

$$
\begin{gathered}
\left\|F_{\frac{i}{A}}^{i}\left(A_{i}, Q_{i}\right)\right\|_{L^{2}\left(I_{T}\right)} \leqslant C_{F}(\mu, \gamma) T^{\alpha}, \\
\left\|F_{\frac{i}{A}}\left(A_{i}^{1}, Q_{i}^{1}\right)-F_{\bar{A}}^{i}\left(A_{i}^{2}, Q_{i}^{2}\right)\right\|_{L^{2}\left(I_{T}\right)} \leqslant C_{F}(\mu, \gamma) T^{\alpha}\left\|\left(A_{i}^{1}, Q_{i}^{1}\right)-\left(A_{i}^{2}, Q_{i}^{2}\right)\right\|_{\mathcal{E}_{T}},
\end{gathered}
$$

for some $\alpha>0$, independent of $T \in(0,1)$, of $\mu \geqslant 1$, and of $\gamma \in(0,1)$.

Proof. Step 1. Proof of (4.17). Let us recall that

$$
\begin{aligned}
& F_{\bar{A}}^{i}\left(A_{i}, Q_{i}\right)=-k_{f} \rho \frac{Q_{i}}{\left(A_{i}\right)^{2}}-\frac{2 \rho Q_{i}}{\left(A_{i}\right)^{2}} \frac{\partial Q_{i}}{\partial x}+\frac{\rho\left(Q_{i}\right)^{2}}{\left(A_{i}\right)^{3}} \frac{\partial A_{i}}{\partial x}-\rho\left(\frac{1}{A_{i}}-\frac{1}{\bar{A}_{i}}\right) \frac{\partial Q_{i}}{\partial t} \\
& +\frac{\partial}{\partial x}\left[\left(\frac{\nu}{2 A_{i, 0} \sqrt{A_{i}}}-\frac{\nu}{2 A_{i, 0} \sqrt{\bar{A}_{i}}}\right) \frac{\partial Q_{i}}{\partial x}\right]-\frac{\beta}{2 A_{i, 0} \sqrt{A_{i}}} \frac{\partial A_{i}}{\partial x}-\frac{1}{4 A_{i, 0} A_{i}^{3 / 2}} \frac{\partial A_{i}}{\partial x} \frac{\partial Q_{i}}{\partial x},
\end{aligned}
$$

- To estimate the first term of $F_{\bar{A}}^{i}$, we write

$$
\left\|-k_{f} \rho \frac{Q_{i}}{\left(A_{i}\right)^{2}}\right\|_{L^{2}\left(I_{T}\right)} \leqslant \frac{k_{f} \rho}{\gamma^{2}}\left\|Q_{i}\right\|_{L^{2}\left(I_{T}\right)} \leqslant C \frac{\sqrt{T}}{\gamma^{2}}\left\|Q_{i}\right\|_{L^{\infty}\left(0, T ; L^{2}(I)\right)} \leqslant C \mu \frac{\sqrt{T}}{\gamma^{2}} .
$$

- The estimate of the second term of $F_{A}^{i}$ is obtained as follows

$$
\left\|\frac{2 \rho Q_{i}}{\left(A_{i}\right)^{2}} \frac{\partial Q_{i}}{\partial x}\right\|_{L^{2}\left(I_{T}\right)} \leqslant C \frac{\mu}{\gamma^{2}}\left\|\frac{\partial Q_{i}}{\partial x}\right\|_{L^{2}\left(I_{T}\right)} \leqslant C \mu \frac{\sqrt{T}}{\gamma^{2}}\left\|\frac{\partial Q_{i}}{\partial x}\right\|_{L^{\infty}\left(0, T ; L^{2}(I)\right)} \leqslant C \mu^{2} \frac{\sqrt{T}}{\gamma^{2}} .
$$

- To estimate the third term of $F_{\bar{A}}^{i}$ (the estimate of sixth term is similar), using (4.11) and (4.14), we have

$$
\left\|\frac{\rho\left(Q_{i}\right)^{2}}{\left(A_{i}\right)^{3}} \frac{\partial A_{i}}{\partial x}\right\|_{L^{2}\left(I_{T}\right)} \leqslant C \frac{\mu^{2}}{\gamma^{3}}\left\|\frac{\partial A_{i}}{\partial x}\right\|_{L^{2}\left(I_{T}\right)} \leqslant C \mu^{3} \frac{\sqrt{T}}{\gamma^{3}} .
$$

- To estimate the fourth term of $F_{\bar{A}}$, with (4.11), we have

$$
\left\|\rho\left(\frac{1}{A_{i}}-\frac{1}{\bar{A}_{i}}\right) \frac{\partial Q_{i}}{\partial t}\right\|_{L^{2}\left(I_{T}\right)} \leqslant C \frac{1}{\gamma^{2}}\left\|A_{i}-\bar{A}_{i}\right\|_{L^{\infty}\left(0, T ; H^{1}(I)\right)}\left\|\frac{\partial Q_{i}}{\partial t}\right\|_{L^{2}\left(I_{T}\right)} \leqslant C \mu^{2} \frac{\sqrt{T}}{\gamma^{2}} .
$$

- To estimate the fifth term of $F_{\bar{A}}^{i}$, with (4.12), we obtain

$$
\begin{aligned}
& \left\|\frac{\partial}{\partial x}\left[\left(\frac{\nu}{2 A_{i, 0} \sqrt{A_{i}}}-\frac{\nu}{2 A_{i, 0} \sqrt{\overline{A_{i}}}}\right) \frac{\partial Q_{i}}{\partial x}\right]\right\|_{L^{2}\left(I_{T}\right)} \\
& \leqslant C \frac{1}{\gamma^{2}}\left\|\sqrt{A}_{i}-\sqrt{\bar{A}_{i}}\right\|_{L^{\infty}\left(0, T ; H^{1}(I)\right)}\left\|\frac{\partial Q_{i}}{\partial x}\right\|_{L^{2}\left(0, T ; H^{1}(I)\right)} \leqslant C \mu^{2} \frac{\sqrt{T}}{\gamma^{5 / 2}} .
\end{aligned}
$$

- To estimate the last term of $F_{\bar{A}}$, using (4.13), we get

$$
\left\|\frac{1}{4 A_{i, 0} A_{i}^{3 / 2}} \frac{\partial A_{i}}{\partial x} \frac{\partial Q_{i}}{\partial x}\right\|_{L^{2}\left(I_{T}\right)} \leqslant C \frac{1}{\gamma^{5 / 2}}\left\|\frac{\partial A_{i}}{\partial x}\right\|_{L^{\infty}\left(0, T ; L^{2}(I)\right)}\left\|\frac{\partial Q_{i}}{\partial x}\right\|_{L^{2}\left(0, T ; H^{s}(I)\right)} \leqslant C \mu^{2} T^{(1-s) / 4} \frac{1}{\gamma^{5 / 2}},
$$

for all $\frac{1}{2}<s<1$.

Step 2. Proof of (4.18). The Lipschitz estimate can be obtained as in Step 1. For clarity let us explain how we can prove it for the fifth term of $F_{\bar{A}}^{i}$ :

$$
\frac{\partial}{\partial x}\left[\left(\frac{\nu}{2 A_{i, 0} \sqrt{A_{i}}}-\frac{\nu}{2 A_{i, 0} \sqrt{\bar{A}_{i}}}\right) \frac{\partial Q_{i}}{\partial x}\right] .
$$


We have

$$
\begin{aligned}
& \frac{\partial}{\partial x}\left[\left(\frac{\nu}{2 A_{i, 0} \sqrt{A_{i}^{1}}}-\frac{\nu}{2 A_{i, 0} \sqrt{\bar{A}_{i}}}\right) \frac{\partial Q_{i}^{1}}{\partial x}\right]-\frac{\partial}{\partial x}\left[\left(\frac{\nu}{2 A_{i, 0} \sqrt{A_{i}^{2}}}-\frac{\nu}{2 A_{i, 0} \sqrt{\bar{A}_{i}}}\right) \frac{\partial Q_{i}^{2}}{\partial x}\right] \\
& =\frac{\partial}{\partial x}\left[\left(\frac{\nu}{2 A_{i, 0} \sqrt{A_{i}^{1}}}-\frac{\nu}{2 A_{i, 0} \sqrt{A_{i}^{2}}}\right) \frac{\partial Q_{i}^{1}}{\partial x}\right]+\frac{\partial}{\partial x}\left[\left(\frac{\nu}{2 A_{i, 0} \sqrt{A_{i}^{2}}}-\frac{\nu}{2 A_{i, 0} \sqrt{\bar{A}_{i}}}\right)\left(\frac{\partial Q_{i}^{1}}{\partial x}-\frac{\partial Q_{i}^{2}}{\partial x}\right)\right] .
\end{aligned}
$$

The first term can be estimated as in Step 1:

$$
\begin{aligned}
& \left\|\frac{\partial}{\partial x}\left[\left(\frac{\nu}{2 A_{i, 0} \sqrt{A_{i}^{1}}}-\frac{\nu}{2 A_{i, 0} \sqrt{A_{i}^{2}}}\right) \frac{\partial Q_{i}^{1}}{\partial x}\right]\right\|_{L^{2}\left(I_{T}\right)} \\
& \leqslant C\left\|\frac{1}{\sqrt{A_{i}^{1}}}-\frac{1}{\sqrt{A_{i}^{2}}}\right\|\left\|_{L^{\infty}\left(0, T ; H^{1}(I)\right)}\right\| \frac{\partial Q_{i}^{1}}{\partial x}\left\|_{L^{2}\left(0, T ; H^{1}(I)\right)}\right\| \frac{\partial Q_{i}^{1}}{\partial x}\left\|_{L^{2}\left(0, T ; H^{1}(I)\right)} \leqslant C \mu \frac{\sqrt{T}}{\gamma^{2}}\right\|\left(A_{i}^{1}, Q_{i}^{1}\right)-\left(A_{i}^{2}, Q_{i}^{2}\right) \|_{\mathcal{E}_{T}} .
\end{aligned}
$$

For the second term, as $\left(A^{2}, Q^{2}\right) \in B_{\bar{A}}(0, T ; \gamma, \mu)$, we have $\left.\left(\sqrt{A_{i}^{2}}-\sqrt{\bar{A}_{i}}\right)\right|_{t=0}=0$. Thus, we can write

$$
\begin{aligned}
& \left\|\frac{\partial}{\partial x}\left[\left(\frac{\nu}{2 A_{i, 0} \sqrt{A_{i}^{2}}}-\frac{\nu}{2 A_{i, 0} \sqrt{\bar{A}_{i}}}\right)\left(\frac{\partial Q_{i}^{1}}{\partial x}-\frac{\partial Q_{i}^{2}}{\partial x}\right)\right]\right\|_{L^{2}\left(I_{T}\right)} \\
& \leqslant C \frac{1}{\gamma^{2}}\left\|\sqrt{A_{i}^{2}}-\sqrt{\bar{A}_{i}}\right\|_{L^{\infty}\left(0, T ; H^{1}(I)\right)}\left\|\frac{\partial Q_{i}^{1}}{\partial x}-\frac{\partial Q_{i}^{2}}{\partial x}\right\|_{L^{2}\left(0, T ; H^{1}(I)\right)} \leqslant C \mu \frac{\sqrt{T}}{\gamma^{5 / 2}}\left\|\left(A_{i}^{1}, Q_{i}^{1}\right)-\left(A_{i}^{2}, Q_{i}^{2}\right)\right\| \|_{\mathcal{E}_{T}} .
\end{aligned}
$$

Estimate of $G_{\bar{A}}^{i}$.

Proposition 4.7. For all $\mu \geqslant 1$ and $\gamma \in(0,1)$, there exists a positive constant $C_{G}(\mu, \gamma)$, depending on $\mu$ and $\gamma$, but independent of $T \in(0,1)$, such that, for all $(A, Q) \in B_{\bar{A}}(0, T ; \gamma, \mu)$, all $\left(A^{1}, Q^{1}\right) \in B_{\bar{A}}(0, T ; \gamma, \mu)$, and all $\left(A^{2}, Q^{2}\right) \in B_{\bar{A}}(0, T ; \gamma, \mu)$, we have

$$
\begin{gathered}
\left\|G_{\bar{A}}^{i}\left(A_{i}, Q_{i}\right)\right\|_{H^{1 / 4}(0, T)} \leqslant C_{G}(\mu, \gamma) T^{\alpha}, \\
\left\|G_{\bar{A}}^{i}\left(A_{i}^{1}, Q_{i}^{1}\right)-G_{\bar{A}}^{i}\left(A_{i}^{2}, Q_{i}^{2}\right)\right\|_{H^{1 / 4}(0, T)} \leqslant C_{G}(\mu, \gamma) T^{\alpha}\left\|\left(A_{i}^{1}, Q_{i}^{1}\right)-\left(A_{i}^{2}, Q_{i}^{2}\right)\right\|_{\mathcal{E}_{T}},
\end{gathered}
$$

for some $\alpha>0$, independent of $T \in(0,1)$, of $\mu \geqslant 1$, and of $\gamma \in(0,1)$.

Proof. Step 1. Proof of (4.19). We only give estimate for $G_{\bar{A}}^{1}$. Let us recall

$$
\begin{aligned}
G \frac{1}{A}\left(A_{1}, Q_{1}\right)=\frac{\beta}{A_{1,0}}\left(\sqrt{A_{1}}(1, t)-\sqrt{\bar{A}_{1}}(1)\right)+\frac{\nu}{2 A_{1,0}} \frac{\partial Q_{1}}{\partial x}(1, t)\left(\frac{1}{\sqrt{\bar{A}_{1}}(1)}-\frac{1}{\sqrt{A_{1}}(1, t)}\right) \\
+\frac{1}{2} \rho \frac{\left(Q_{1}\right)^{2}(1, t)}{\left(A_{1}\right)^{2}(1, t)} .
\end{aligned}
$$

- Estimate of first term of $G_{\frac{1}{A}}^{1}$. We apply Lemma $4.2(i)$ with $s_{1}=1 / 4, s_{2}=1$ and $g=\sqrt{A_{1}}(1, \cdot)-\sqrt{\bar{A}_{1}}(1)$ and relation (4.15), and we obtain

$$
\begin{aligned}
\left\|\sqrt{A_{1}}(1, \cdot)-\sqrt{\bar{A}_{1}}(1)\right\|_{H^{1 / 4}(0, T)} \leqslant C T^{3 / 8}\left\|\sqrt{A_{1}}(1, \cdot)-\sqrt{\bar{A}_{1}}(1)\right\|_{H^{1}(0, T)} & \leqslant C T^{3 / 8}\left\|\sqrt{A_{1}}-\sqrt{\bar{A}_{1}}\right\|_{H^{1}\left(0, T ; H^{1}(I)\right)} \leqslant C \mu T^{7 / 8} .
\end{aligned}
$$


- Estimate of the second term of $G_{\bar{A}}^{1}$. We apply Lemma $4.2(i i i)$ with $s=1 / 4, s_{1}=1 / 4, s_{2}=1, f=\frac{\partial Q_{1}}{\partial x}(1, \cdot)$, $g=\sqrt{A_{1}}(1, \cdot)-\sqrt{\bar{A}_{1}}(1)$ and Lemma 4.4 , and we have

$$
\begin{gathered}
\left\|\frac{\partial Q_{1}}{\partial x}(1, \cdot)\left(\frac{1}{\sqrt{\bar{A}_{1}}(1)}-\frac{1}{\sqrt{A_{1}}(1, \cdot)}\right)\right\|_{H^{1 / 4}(0, T)} \leqslant C\left\|\frac{\partial Q_{1}}{\partial x}(1, \cdot)\left(\sqrt{\bar{A}_{1}}(1)-\sqrt{A_{1}}(1, \cdot)\right)\right\|_{H^{1 / 4}(0, T)} \\
\leqslant C T^{\alpha}\left(\left|\frac{\partial Q_{1}}{\partial x}(1,0)\right|+\left\|\frac{\partial Q_{1}}{\partial x}(1, \cdot)\right\|\right)_{H^{1 / 4}(0, T)}\left\|\sqrt{\bar{A}_{1}}(1)-\sqrt{A_{1}}(1, \cdot)\right\|_{H^{1}(0, T)} \leqslant C \mu^{2} T^{\alpha} .
\end{gathered}
$$

- Estimate of the last term in $G_{\bar{A}}^{1}$. We rewrite the last term of $G_{\bar{A}}^{1}$ as follows

$$
\frac{1}{2} \rho \frac{\left(Q_{1}\right)^{2}}{\left(A_{1}\right)^{2}}(1, t)=\frac{\rho}{2}\left(\left(Q_{1}\right)^{2}\left(\frac{1}{\left(A_{1}\right)^{2}}-\frac{1}{\left(\bar{A}_{1}\right)^{2}}\right)(1, t)+\frac{\left(\left(Q_{1}\right)^{2}(1, t)-\left|Q_{1}^{0}\right|^{2}(1)\right)}{\left(\bar{A}_{1}\right)^{2}(1)}+\frac{\left|Q_{1}^{0}\right|^{2}(1)}{\left(\bar{A}_{1}\right)^{2}(1)}\right) .
$$

First of all, using [12, Proposition B1], for any $s_{0} \in(1 / 8,1 / 4)$, we have

$$
\left\|\left(Q_{1}\right)^{2}(1, \cdot)\right\|_{H^{1 / 4}(0, T)} \leqslant C\left\|Q_{1}(1, \cdot)\right\|_{H^{1 / 4+s_{0}(0, T)}}^{2} \leqslant C\left\|Q_{1}(1, \cdot)\right\|_{H^{3 / 4}(0, T)}^{2},
$$

where the constant $C$ can be chosen independent of $T$. Indeed, as $1 / 4+s_{0}<1 / 2$, we can extend the function $Q_{1}(1, t)$ by zero to $(T, \infty)$ and the estimate follows easily. Using Lemma 4.4 in the above estimate, we obtain

$$
\left\|\left(Q_{1}\right)^{2}(1, \cdot)\right\|_{H^{1 / 4}(0, T)} \leqslant C \mu^{2},
$$

with the constant $C$ is independent of $T$. Therefore, using Lemma 4.2 and relation (4.16) of Proposition 4.5, we have

$$
\begin{aligned}
& \left\|\left(Q_{1}\right)^{2}\left(\frac{1}{\left(A_{1}\right)^{2}}-\frac{1}{\left(\bar{A}_{1}\right)^{2}}\right)(1, \cdot)\right\|_{H^{1 / 4}(0, T)} \leqslant C\left\|\left(Q_{1}\right)^{2}(1, \cdot)\right\|_{H^{1 / 4}(0, T)}\left\|\frac{1}{\left(A_{1}\right)^{2}(1, \cdot)}-\frac{1}{\left(\bar{A}_{1}\right)^{2}(1)}\right\|_{H^{1}(0, T)} \\
& \leqslant C T^{\alpha} \mu^{2}\left\|\left(A_{i}\right)^{2}-\left(\bar{A}_{1}\right)^{2}\right\|_{H^{1}\left(0, T ; H^{1}(I)\right)} \leqslant C T^{\alpha} \mu^{4} .
\end{aligned}
$$

To estimate second term of (4.21), we note that, by using Lemma 4.2 , for any $s_{0} \in(1 / 8,1 / 4)$, we have

$$
\begin{aligned}
& \left\|\frac{\left(Q_{1}\right)^{2}(1, \cdot)-\left|Q_{1}^{0}\right|^{2}(1)}{\left(\bar{A}_{1}\right)^{2}(1)}\right\|_{H^{1 / 4}(0, T)} \\
& \left.\leqslant C \| Q_{1}(1, \cdot)+Q_{1}^{0}(1)\right)\left\|_{H^{1 / 4+s_{0}}(0, T)}\right\| Q_{1}(1, t)-Q_{1}^{0}(1) \|_{H^{1 / 4+s_{0}}(0, T)} \\
& \leqslant C \mu T^{\alpha},
\end{aligned}
$$

for some $C$ independent of $T$ and $\alpha>0$.

The last term of (4.21) can be estimated as follows

$$
\left\|\frac{\left|Q_{1}^{0}\right|^{2}(1)}{\left(\bar{A}_{1}\right)^{2}(1)}\right\|_{H^{1 / 4}(0, T)} \leqslant \frac{\left|Q_{1}^{0}(1)\right|^{2}}{\gamma^{2}} \sqrt{T} \leqslant C \sqrt{T} .
$$

Step 2. The Lipschitz estimate (4.20) can be obtained as in Step 1.

In Section 6, we are going to consider nonlinear Robin boundary conditions of the form

$$
-\frac{\nu}{2 A_{1,0} \sqrt{\bar{A}_{1}}} \frac{\partial Q_{1}}{\partial x}(0, t)+Q_{1}(0, t)=H_{1}\left(Q_{1}, A_{1}\right)-h_{1}(t),
$$

and for $i=1,2$,

$$
\frac{\nu}{2 A_{i, 0} \sqrt{\bar{A}_{1}}} \frac{\partial Q_{i}}{\partial x}(0, t)+Q_{i}(0, t)=H_{i}\left(Q_{i}, A_{i}\right)-h_{i}(t),
$$


with

$$
H_{\bar{A}}\left(Q_{1}, A_{1}\right)=\left.\left[-\frac{\beta}{A_{1,0}}\left(\sqrt{A_{1}}-\sqrt{\bar{A}_{1}}\right)+\frac{\nu}{2 A_{1,0}} \frac{\partial Q_{1}}{\partial x}\left(\frac{1}{\sqrt{A_{1}}}-\frac{1}{\sqrt{\bar{A}_{1}}}\right)-\frac{1}{2} \rho \frac{Q_{1}^{2}}{A_{1}^{2}}\right]\right|_{x=1},
$$

and for $i=1,2$,

$$
H \frac{i}{A}\left(Q_{i}, A_{i}\right)=\left.\left[\frac{\beta}{A_{i, 0}}\left(\sqrt{A_{i}}-\sqrt{\bar{A}_{i}}\right)-\frac{\nu}{2 A_{i, 0}} \frac{\partial Q_{i}}{\partial x}\left(\frac{1}{\sqrt{A_{i}}}-\frac{1}{\sqrt{\bar{A}_{i}}}\right)+\frac{1}{2} \rho \frac{Q_{i}^{2}}{A_{i}^{2}}\right]\right|_{x=0} .
$$

Estimate of $H_{\frac{i}{A}}$. To study (1.8) in which the Dirichlet boundary condition (1.8) $)_{5}$ are replaced by the nonlinear Robin boundary conditions stated in (4.22), we have to estimate the nonlinear terms defined in (4.23).

Proposition 4.8. For all $\mu \geqslant 1$ and $\gamma \in(0,1)$, there exists a positive constant $C_{H}(\mu, \gamma)$, depending on $\mu$ and $\gamma$, but independent of $T \in(0,1)$, such that, for all $(A, Q) \in B_{\bar{A}}(0, T ; \gamma, \mu)$, all $\left(A^{1}, Q^{1}\right) \in B_{\bar{A}}(0, T ; \gamma, \mu)$, and all $\left(A^{2}, Q^{2}\right) \in B_{\bar{A}}(0, T ; \gamma, \mu)$, we have

$$
\begin{gathered}
\left\|H_{\bar{A}}^{i}\left(A_{i}, Q_{i}\right)\right\|_{H^{1 / 4}(0, T)} \leqslant C_{H}(\mu, \gamma) T^{\alpha}, \\
\left\|H_{\frac{i}{A}}\left(A_{i}^{1}, Q_{i}^{1}\right)-H_{\frac{i}{A}}\left(A_{i}^{2}, Q_{i}^{2}\right)\right\|_{H^{1 / 4}(0, T)} \leqslant C_{H}(\mu, \gamma) T^{\alpha}\left\|\left(A_{i}^{1}, Q_{i}^{1}\right)-\left(A_{i}^{2}, Q_{i}^{2}\right)\right\|_{\mathcal{E}_{T}},
\end{gathered}
$$

for some $\alpha>0$, independent of $T \in(0,1)$, of $\mu \geqslant 1$, and of $\gamma \in(0,1)$.

Proof. Step 1. Proof of (4.24). We only give estimate for $H_{\frac{1}{A}}$. Let us recall that

$$
H \frac{1}{A}\left(Q_{1}, A_{1}\right)=\left.\left[-\frac{\beta}{A_{1,0}}\left(\sqrt{A_{1}}-\sqrt{\bar{A}_{1}}\right)+\frac{\nu}{2 A_{1,0}} \frac{\partial Q_{1}}{\partial x}\left(\frac{1}{\sqrt{A_{1}}}-\frac{1}{\sqrt{A_{1}}}\right)-\frac{1}{2} \rho \frac{Q_{1}^{2}}{A_{1}^{2}}\right]\right|_{x=1} .
$$

Using the relation (4.12) of Proposition 4.5, we obtain

$$
\left\|\sqrt{A_{1}}(1, \cdot)-\sqrt{\bar{A}_{1}}(1)\right\|_{H^{1 / 4}(0, T)} \leqslant\left\|\sqrt{A_{1}}(1, \cdot)-\sqrt{\bar{A}_{1}}(1)\right\|_{H^{1}(0, T)} \leqslant \frac{C \mu}{\sqrt{\gamma}} T^{1 / 2}
$$

The term $\left.\left[\frac{\partial Q_{1}}{\partial x}\left(\frac{1}{\sqrt{A_{1}}}-\frac{1}{\sqrt{\bar{A}_{1}}}\right)\right]\right|_{x=1}$ can be estimated as follows

$$
\begin{aligned}
& \left\|\frac{\partial Q_{1}}{\partial x}(1, \cdot)\left(\frac{1}{\sqrt{A_{1}}(1, \cdot)}-\frac{1}{\sqrt{\overline{A_{1}}(1)}}\right)\right\|_{H^{1 / 4}(0, T)}\left\|\frac{1}{\sqrt{A_{1}}(1, \cdot)}-\frac{1}{\sqrt{\bar{A}_{1}}(1)}\right\|_{H^{1}(0, T)} \\
& \leqslant\left\|\frac{\partial Q_{1}}{\partial x}(1, \cdot)\right\|_{H^{1 / 4}(0, T)} \| C \frac{\mu}{\gamma^{3 / 2}} \sqrt{T} .
\end{aligned}
$$

The estimate of the other term can be obtained similarly.

Step 2. The Lipschitz estimate (4.25) can be obtained as in Step 1.

\section{EXISTENCE AND UNIQUENESS OF MAXIMAL SOLUTION}

5.1. Proof of Theorem 1.2. Proof. Step 1. Existence of a local-in-time strong solution. We choose $M>0$ and $\mu>0$ such that

$$
\sum_{i=1}^{3}\left(\left\|A_{i}^{0}\right\|_{H^{1}(I)}+\left\|Q_{i}^{0}\right\|_{H^{1}(I)}+\left|g_{A^{0}}^{i}\right|+\left\|h_{i}\right\|_{H^{3 / 4}(0,1)}\right) \leqslant M \quad \text { and } \quad \mu=2 C_{A^{0}} M,
$$

where $C_{A^{0}}$ is the continuity constant appearing in (3.14) corresponds to $\bar{A}=A^{0}$. Let us set

$$
\gamma=\frac{1}{2} \gamma_{A^{0}} \text { with } \gamma_{A^{0}}=\min \left\{A_{1}^{0}(x), A_{2}^{0}(x), A_{3}^{0}(x) \mid x \in I\right\}>0 .
$$


Let $\left(\psi_{i}, \Phi_{i}\right)_{i=1}^{3}$ belong to $B_{A^{0}}(0, T ; \gamma, \mu)$ (see (4.10)). We consider the following system:

For $i \in\{1,2,3\},\left(A_{i}, Q_{i}\right)$ satisfies

$$
\begin{aligned}
& \frac{\partial A_{i}}{\partial t}+\frac{\partial Q_{i}}{\partial x}=0, \quad t \in(0, T), x \in I, \\
& L_{A^{0}}^{i} \frac{\partial Q_{i}}{\partial t}-\frac{\partial}{\partial x}\left(N_{A^{0}}^{i} \frac{\partial Q_{i}}{\partial x}\right)=F_{A^{0}}^{i}\left(\psi_{i}, \Phi_{i}\right), \quad t \in(0, T), \quad x \in I, \\
& Q_{1}(1, t)=Q_{2}(0, t)+Q_{3}(0, t), \quad t \in(0, T), \\
& Q_{1}(0, t)=h_{1}(t), \quad Q_{2}(1, t)=h_{2}(t), \quad Q_{3}(1, t)=h_{3}(t), \quad t \in(0, T), \\
& -N_{A^{0}}^{1} \frac{\partial Q_{1}}{\partial x}(1, t)+g_{A^{0}}^{1}+G_{A^{0}}^{1}\left(\psi_{1}, \Phi_{1}\right)=-N_{A^{0}}^{2} \frac{\partial Q_{2}}{\partial x}(0, t)+g_{A^{0}}^{2}+G_{A^{0}}^{2}\left(\psi_{2}, \Phi_{2}\right) \\
& \quad=-N_{A^{0}}^{3} \frac{\partial Q_{3}}{\partial x}(0, t)+g_{A^{0}}^{3}+G_{A^{0}}^{3}\left(\psi_{3}, \Phi_{3}\right), \quad t \in(0, T), \\
& A_{i}(x, 0)=A_{i}^{0}(x), \quad Q_{i}(x, 0)=Q_{i}^{0}(x), \quad x \in I .
\end{aligned}
$$

We are going to show that, there exists $0<T \leqslant 1$ such that the mapping

$$
\mathcal{N}:\left(\psi_{i}, \Phi_{i}\right)_{i=1}^{3} \mapsto\left(A_{i}, Q_{i}\right)_{i=1}^{3},
$$

where $\left(A_{i}, Q_{i}\right)_{i=1}^{3}$ is the solution to system $(5.2)$, is a strict contraction in $B_{A^{0}}(0, T ; \gamma, \mu)$.

Applying Theorem 3.7 to system (5.2), we obtain

$$
\begin{aligned}
\left\|\left(A_{i}, Q_{i}\right)_{i=1}^{3}\right\| \|_{\mathcal{E}(0, T)} \leqslant C_{A^{0}} \sum_{i=1}^{3}\left(\left\|F_{A^{0}}^{i}\left(\psi_{i}, \Phi_{i}\right)\right\|_{L^{2}\left(0, T ; L^{2}(I)\right)}\right. & +\left\|G_{A^{0}}^{i}\left(\psi_{i}, \Phi_{i}\right)\right\|_{H^{1 / 4}(0, T)}+\left|g_{A^{0}}^{i}\right| \\
& \left.+\left\|h_{i}\right\|_{H^{3 / 4}(0, T)}+\left\|A_{i}^{0}\right\|_{H^{1}(I)}+\left\|Q_{i}^{0}\right\|_{H^{1}(I)}\right) .
\end{aligned}
$$

Since $\left(\psi_{i}, \Phi_{i}\right) \in B_{A^{0}}(0, T ; \gamma, \mu)$, applying Proposition 4.6, Proposition 4.7 and relation (5.1), estimate (5.3) becomes

$$
\left\|\left(A_{i}, Q_{i}\right)_{i=1}^{3}\right\|_{\mathcal{E}(0, T)} \leqslant C_{A^{0}} M+C_{A^{0}}\left(C_{F}+C_{G}\right) T^{\alpha} \quad \text { for all } 0<T \leqslant 1 .
$$

Therefore, with the choice of $\mu$ in relation (5.1), there exists $T>0$ small enough such that

$$
\left\|\mathcal{N}\left(\left(\psi_{i}, \Phi_{i}\right)_{i=1}^{3}\right)\right\| \|_{\mathcal{E}(0, T)} \leqslant \mu \text {. }
$$

Using the continuous embedding $L^{\infty}\left(0, T ; H^{1}(I)\right) \hookrightarrow L^{\infty}\left(I_{T}\right)$, from (4.11) we obtain

$$
\left\|A_{i}-A_{i}^{0}\right\|_{L^{\infty}\left(I_{T}\right)} \leqslant C \sqrt{T}
$$

with $C$ independent of $T$ since $A_{i}(0)=A_{i}^{0}$. By choosing $T$ small enough, we get $A_{i}(x, t) \geqslant \gamma$ for all $(x, t) \in I_{T}$ because $\gamma=\gamma_{A^{0}} / 2$. Therefore, $\mathcal{N}$ maps $B_{A^{0}}(0, T ; \gamma, \mu)$ into itself.

Now we will show that $\mathcal{N}$ is a contraction. Let $\left(\psi_{i}^{1}, \Phi_{i}^{1}\right)_{i=1}^{3}$ and $\left(\psi_{i}^{2}, \Phi_{i}^{2}\right)_{i=1}^{3}$ belong to $B_{A^{0}}(0, T ; \gamma, \mu)$. Using Theorem 3.7, Propositions 4.6 and 4.7, we obtain

$$
\begin{aligned}
& \|\| \mathcal{N}\left(\left(\psi_{i}^{1}, \Phi_{i}^{1}\right)_{i=1}^{3}\right)-\mathcal{N}\left(\left(\psi_{i}^{2}, \Phi_{i}^{2}\right)_{i=1}^{3}\right) \|_{\mathcal{E}(0, T)} \\
& \leqslant C_{A^{0}}\left(C_{F}+C_{G}\right) T^{\alpha}\left\|\left(\psi_{i}^{1}, \Phi_{i}^{1}\right)_{i=1}^{3}-\left(\psi_{i}^{2}, \Phi_{i}^{2}\right)_{i=1}^{3}\right\|_{\mathcal{E}(0, T)} .
\end{aligned}
$$

Thus $\mathcal{N}$ is a contraction in $B_{A^{0}}(0, T ; \gamma, \mu)$ for $T$ small enough. The proof of the existence of $T \in(0,1]$, for which the system (1.8) admits at least one strong solution over $[0, T]$, is complete.

Step 2. Existence of a maximal solution. Let us prove that any local-in-time strong solution may be extended to a maximal strong solution. Let $(A, Q)$ be a local-in-time solution to $(1.8)$ over $\left[0, T_{1}\right]$. We want to show that $(A, Q)$ can be extended as a maximal strong solution over $\left[0, T_{m}\right)$, with $T_{m}>T_{1}$. We look for $(\widehat{A}, \widehat{Q}, T)$, with $T \geqslant T_{1}$ and $(\widehat{A}, \widehat{Q}) \in \mathcal{E}(0, T)$, such that

$$
\begin{aligned}
& (\widehat{A}, \widehat{Q})(t)=(A, Q)(t) \text { for all } t \in\left[0, T_{1}\right], \\
& (\widehat{A}, \widehat{Q}) \text { is solution to }(1.8) \text { over }[0, T] .
\end{aligned}
$$


The set of triplets $(\widehat{A}, \widehat{Q}, T)$ satisfying (5.4) is nonempty since $\left(A, Q, T_{1}\right)$ satisfies (5.4). For a given triplet $(\widehat{A}, \widehat{Q}, T)$ satisfying $(5.4)$, we set

$$
\widehat{T}=\sup \left\{T \geqslant T_{1} \mid(\widehat{A}, \widehat{Q}, T) \text { satisfies (5.4) }\right\}
$$

If $\widehat{T}=\infty$, then the proof is complete.

Let us assume that $\widehat{T}<\infty$, and that $(\widehat{A}, \widehat{Q}, T)$ satisfies (5.4) for all $T<\widehat{T}<\infty$. We have to show that (1.15) holds for $(A, Q)=(\widehat{A}, \widehat{Q})$ and $T_{m}=\widehat{T}$. We argue by contradiction. We assume that

$$
\lim _{T \rightarrow \widehat{T}}\left(\|(\widehat{A}, \widehat{Q})\|_{\mathcal{E}(0, T)}+\max \left\{\left|\widehat{A}_{i}(x, T)\right|^{-1} \mid 1 \leqslant i \leqslant 3, x \in I\right\}\right)<\infty .
$$

Let us set

$$
\|(\widehat{A}, \widehat{Q})\|_{\mathcal{E}(0, \widehat{T})}=\mu_{\widehat{T}} \text { and } \sup \left\{\left|\widehat{A}_{i}(x, T)\right| \mid 1 \leqslant i \leqslant 3, x \in I, T \in[0, \widehat{T})\right\}=\gamma_{\widehat{T}}>0 .
$$

We are going to show that the solution $(\widehat{A}, \widehat{Q})$ can be extended to $[\widehat{T}, \widehat{T}+\varepsilon]$, for some $\varepsilon>0$, so that $(\widehat{A}, \widehat{Q})$ is solution to system $(1.8)$ over the time interval $[0, \widehat{T}+\varepsilon]$.

We set $\bar{A}=A(x, \widehat{T}), x \in I$. We consider the system over the time interval $(\widehat{T}, \tau)$ with $\tau>\widehat{T}$ :

$$
\begin{aligned}
& \text { For } i \in\{1,2,3\},\left(\widehat{A}_{i}, \widehat{Q}_{i}\right) \text { satisfies } \\
& \frac{\partial \widehat{A}_{i}}{\partial t}+\frac{\partial \widehat{Q}_{i}}{\partial x}=0, \quad t \in(\widehat{T}, \tau), x \in I, \\
& L_{\frac{i}{A}} \frac{\partial \widehat{Q}_{i}}{\partial t}-\frac{\partial}{\partial x}\left(N \frac{i}{A} \frac{\partial \widehat{Q}_{i}}{\partial x}\right)=F \frac{i}{A}\left(\widehat{A}_{i}, \widehat{Q}_{i}\right), \quad t \in(\widehat{T}, \tau), \quad x \in I, \\
& \widehat{Q}_{1}(1, t)=\widehat{Q}_{2}(0, t)+\widehat{Q}_{3}(0, t), \quad t \in(\widehat{T}, \tau), \\
& \widehat{Q}_{1}(0, t)=h_{1}(t), \quad \widehat{Q}_{2}(1, t)=h_{2}(t), \quad \widehat{Q}_{3}(1, t)=h_{3}(t), \quad t \in(\widehat{T}, \tau), \\
& -N_{\frac{1}{A}} \frac{\partial \widehat{Q}_{1}}{\partial x}(1, t)+g_{\frac{1}{A}}+G_{\frac{1}{A}}\left(\widehat{A}_{1}, \widehat{Q}_{1}\right)=-N_{\frac{2}{A}} \frac{\partial \widehat{Q}_{2}}{\partial x}(0, t)+g_{\frac{2}{A}}+G_{\frac{2}{A}}\left(\widehat{A}_{2}, \widehat{Q}_{2}\right) \\
& \quad=-N_{\frac{3}{A}} \frac{\partial \widehat{Q}_{3}}{\partial x}(0, t)+g_{\frac{3}{A}}+G_{\frac{3}{A}}\left(\widehat{A}_{3}, \widehat{Q}_{3}\right), \quad t \in(\widehat{T}, \tau), \\
& \widehat{A}_{i}(x, \widehat{T})=A_{i}(x, \widehat{T}), \quad \widehat{Q}_{i}(x, \widehat{T})=Q_{i}(x, \widehat{T}), \quad x \in I,
\end{aligned}
$$

where the coefficients $L_{\bar{A}}^{i}, N_{\bar{A}}^{i}$ and the nonlinear terms $F_{\bar{A}}^{i}, G_{\bar{A}}^{i}, g_{\bar{A}}^{i}$ are defined by (2.1)-(2.3). We consider the system

$$
\begin{aligned}
& \frac{\partial \widehat{A}_{i}}{\partial t}+\frac{\partial \widehat{Q}_{i}}{\partial x}=0, \quad t \in(\widehat{T}, \tau), x \in I, i \in\{1,2,3\} \\
& L_{\bar{A}}^{i} \frac{\partial \widehat{Q}_{i}}{\partial t}-\frac{\partial}{\partial x}\left(N_{\bar{A}} \frac{\partial \widehat{Q}_{i}}{\partial x}\right)=F_{\bar{A}}^{i}\left(\psi_{i}, \Phi_{i}\right), \quad t \in(\widehat{T}, \tau), x \in I, i \in\{1,2,3\} \\
& \widehat{Q}_{1}(1, t)=\widehat{Q}_{2}(0, t)+\widehat{Q}_{3}(0, t), \quad t \in(\widehat{T}, \tau), \\
& \widehat{Q}_{1}(0, t)=h_{1}(t), \quad \widehat{Q}_{2}(1, t)=h_{2}(t), \quad \widehat{Q}_{3}(1, t)=h_{3}(t), \quad t \in(\widehat{T}, \tau), \\
& -N_{\frac{1}{A}} \frac{\partial \widehat{Q}_{1}}{\partial x}(1, t)+g_{\frac{1}{A}}+G_{\bar{A}}\left(\psi_{1}, \Phi_{1}\right)=-N_{\bar{A}} \frac{\partial \widehat{Q}_{2}}{\partial x}(0, t)+g_{\bar{A}}^{2}+G_{\bar{A}}^{2}\left(\psi_{2}, \Phi_{2}\right) \\
& \quad=-N_{\frac{3}{A}} \frac{\partial \widehat{Q}_{3}}{\partial x}(0, t)+g_{\bar{A}}^{3}+G_{\bar{A}}^{3}\left(\psi_{3}, \Phi_{3}\right), \quad t \in(\widehat{T}, \tau), \\
& \widehat{A}_{i}(x, \widehat{T})=A_{i}(x, \widehat{T}), \quad \widehat{Q}_{i}(x, \widehat{T})=Q_{i}(x, \widehat{T}), \quad x \in I, i \in\{1,2,3\} .
\end{aligned}
$$

We set

$$
\widetilde{\gamma}=\gamma_{\widehat{T}} / 2 \text { and } \widetilde{\mu}=\widehat{C}_{\bar{A}} \mu_{\widehat{T}}+\mu_{\widehat{T}}
$$


For $\tau>\widehat{T}$, we define the mapping $\widetilde{\mathcal{N}}_{\tau}$ in the ball $B_{\bar{A}}(\widehat{T}, \tau ; \widetilde{\gamma}, \widetilde{\mu})$ by

$$
\widetilde{\mathcal{N}}_{\tau}:\left(\psi_{i}, \Phi_{i}\right)_{i=1}^{3} \mapsto\left(\widehat{A}_{i}, \widehat{Q}_{i}\right)_{i=1}^{3},
$$

where $\left(\widehat{A}_{i}, \widehat{Q}_{i}\right)_{i=1}^{3}$ is the solution to system (5.7) over $[\widehat{T}, \tau]$, and $B_{\bar{A}}(\widehat{T}, \tau ; \widetilde{\gamma}, \widetilde{\mu})$ is defined in (4.10).

Applying Theorem 3.7 to system (5.7), as in Step 1, we can show that $\widetilde{\mathcal{N}}_{\tau}$ is a contraction in $B_{\bar{A}}(\widehat{T}, \tau ; \widetilde{\gamma}, \widetilde{\mu})$ for $\tau-\widehat{T}>0$ small enough. Thus the system (5.6) admits a solution over the time interval $[\widehat{T}, \tau]$ and the system (1.8) admits a solution over the time interval $[0, \tau]$. We have a contradiction with the definition of $\widehat{T}$. Thus $(5.5)$ is false and (1.15) is proved. Hence, we have proved that any local-in-time strong solution may be extended to a maximal strong solution.

Step 3. Uniqueness of maximal solution. Let us prove that system (1.8) admits a unique maximal solution. Let $(A, Q)$ be a maximal solution to system $(1.8)$ over $\left[0, T_{\mathrm{m}}\right)$, and let $(\widetilde{A}, \widetilde{Q})$ be another maximal solution to system $(1.8)$ over $\left[0, \widetilde{T}_{\mathrm{m}}\right)$. Let us assume that $T_{\mathrm{m}} \leqslant \widetilde{T}_{\mathrm{m}}$. Let us set

$$
\widehat{T}_{\mathrm{m}}=\sup \left\{t \in\left[0, T_{\mathrm{m}}\right) \mid(A, Q)(\tau)=(\widetilde{A}, \widetilde{Q})(\tau) \text { for all } \tau \in[0, t]\right\} .
$$

If $\widehat{T}_{\mathrm{m}}=T_{\mathrm{m}}=\widetilde{T}_{\mathrm{m}}$, then the two maximal solutions are identical and the proof is complete.

If $\widehat{T}_{\mathrm{m}}=T_{\mathrm{m}}<\widetilde{T}_{\mathrm{m}}$, then

$$
\begin{aligned}
& \lim _{T \rightarrow T_{\mathrm{m}}}\left(\|(A, Q)\|_{\mathcal{E}(0, T)}+\max \left\{\left|A_{i}(x, T)\right|^{-1} \mid 1 \leqslant i \leqslant 3, x \in[0,1]\right\}\right) \\
& =\left(\|(\widetilde{A}, \widetilde{Q})\|_{\left.{\mathcal{E}\left(0, T_{\mathrm{m}}\right)}+\max \left\{\left|A_{i}\left(x, T_{\mathrm{m}}\right)\right|^{-1} \mid 1 \leqslant i \leqslant 3, x \in[0,1]\right\}\right)<\infty,}\right.
\end{aligned}
$$

which is in contradiction with the fact that $(A, Q)$ is a maximal solution to system (1.8) over $\left[0, T_{\mathrm{m}}\right)$. Thus the proof is complete in that case too.

Let us examine the last case $\widehat{T}_{\mathrm{m}}<T_{\mathrm{m}}$. We have to treat separately the cases when $\widehat{T}_{\mathrm{m}}>0$ and the case when $\widehat{T}_{\mathrm{m}}=0$. The case when $\widehat{T}_{\mathrm{m}}=0$ can be treated with the same arguments as in Step 1. Let us treat the case when $\widehat{T}_{\mathrm{m}}>0$. We set

$$
\begin{aligned}
& \mu=\|(A, Q)\|_{\mathcal{E}\left(0, \widehat{T}_{\mathrm{m}}\right)}, \quad \gamma=\min \left\{A_{i}(x, t) \mid 1 \leqslant i \leqslant 3, x \in I, t \in\left[0, \widehat{T}_{\mathrm{m}}\right]\right\}, \\
& \widetilde{\mu}=\|(\widetilde{A}, \widetilde{Q})\|_{\mathcal{E}\left(0, \widehat{T}_{\mathrm{m}}\right)}, \quad \widetilde{\gamma}=\min \left\{\widetilde{A}_{i}(x, t) \mid 1 \leqslant i \leqslant 3, x \in I, t \in\left[0, \widehat{T}_{\mathrm{m}}\right]\right\}, \\
& \widehat{\mu}=2 \mu+2 \widetilde{\mu}, \quad \text { and } \hat{\gamma}=\min (\gamma / 2, \widetilde{\gamma} / 2) .
\end{aligned}
$$

Now we set $\bar{A}=A\left(\widehat{T}_{\mathrm{m}}\right)$. For $\widehat{T}_{\mathrm{m}}<\tau<T_{\mathrm{m}}$, we notice that the function $(\widehat{A}, \widehat{Q})=(A, Q)-(\widetilde{A}, \widetilde{Q})$ satisfies the linear system $(3.1)$ over $\left[\widehat{T}_{\mathrm{m}}, \tau\right]$, with

$$
\begin{aligned}
& f_{i}=F_{\bar{A}}^{i}\left(A_{i}, Q_{i}\right)-F_{\bar{A}}^{i}\left(\widetilde{A}_{i}, \widetilde{Q}_{i}\right), \\
& g_{i}=G_{\bar{A}}^{i}\left(A_{i}, Q_{i}\right)-G i \frac{i}{A}\left(\widetilde{A}_{i}, \widetilde{Q}_{i}\right), \\
& h_{i}=0, \widehat{A}_{i}\left(x, \widehat{T}_{\mathrm{m}}\right)=0, \quad \widehat{Q}_{i}\left(x, \widehat{T}_{\mathrm{m}}\right)=0 .
\end{aligned}
$$

Using Theorem 3.7, Proposition 4.6 and Proposition 4.7, we obtain

$$
\|(\widehat{A}, \widehat{Q})\|_{\mathcal{E}\left(\widehat{T}_{\mathrm{m}}, \tau\right)} \leqslant C_{\bar{A}}\left(C_{F}+C_{G}\right)\left(\tau-\widehat{T}_{\mathrm{m}}\right)^{\alpha}\|(\widehat{A}, \widehat{Q})\|_{\mathcal{E}\left(\widehat{T}_{\mathrm{m}}, \tau\right)} .
$$

We choose $\widehat{T}_{\mathrm{m}}<\tau<T_{\mathrm{m}}$ such that $\tau-\widehat{T}_{\mathrm{m}}$ is small enough to have $C_{\bar{A}}\left(C_{F}+C_{G}\right)\left(\tau-\widehat{T}_{\mathrm{m}}\right)^{\alpha} \leqslant 1 / 2$. From the above estimate we deduce that $\|(\widehat{A}, \widehat{Q})\|_{\mathcal{E}\left(\widehat{T}_{\mathrm{m}}, \tau\right)}=0$. Thus $(A, Q)(t)=(\widetilde{A}, \widetilde{Q})(t)$ for all $t \in[0, \tau]$. We have a contradiction with the definition of $\widehat{T}_{\mathrm{m}}$, and the proof is complete. 
5.2. Maximal solution for the $(A, u)$-system. We end this section by reformulating the system $(1.4)-($ in terms of the independent variables $A(x, t)$, the cross sectional area, and $u(x, t):=\frac{Q(x, t)}{A(x, t)}$, the average fluid velocity. Moreover, we prove an existence and uniqueness result for the system written in variables $A$ and $u$.

The velocity $u_{i}(x, t)$ on the $i$-th vessel is

$$
u_{i}(x, t)=\frac{Q_{i}(x, t)}{A_{i}(x, t)}, \quad x \in I, t \in(0, T) .
$$

Then the system $(1.4)-(1.7)$ can be written as:

$$
\left\{\begin{array}{l}
\text { For } i \in\{1,2,3\},\left(A_{i}, u_{i}\right) \text { satisfies } \\
\frac{\partial A_{i}}{\partial t}+\frac{\partial}{\partial x}\left(A_{i} u_{i}\right)=0, \quad t \in(0, T), x \in I, \\
\frac{\partial}{\partial t}\left(A_{i} u_{i}\right)+\frac{\partial}{\partial x}\left(A_{i} u_{i}^{2}\right)+\frac{A_{i}}{\rho} \frac{\partial P_{i}}{\partial x}+k_{f} u_{i}=0, \quad t \in(0, T), x \in I, \\
P_{i}=P_{\text {ext }}+\frac{\beta}{A_{i, 0}}\left(\sqrt{A_{i}}-\sqrt{A_{i, 0}}\right)+\frac{\nu}{A_{i, 0}} \frac{\partial}{\partial t}\left(\sqrt{A_{i}}\right), \\
A_{i}(x, 0)=A_{i}^{0}(x), \quad u_{i}(x, 0)=u_{i}^{0}(x), \quad x \in I,
\end{array}\right.
$$

with the following nonlinear boundary conditions at the branching points

$$
\left\{\begin{array}{l}
A_{1} u_{1}(1, t)=A_{2} u_{2}(0, t)+A_{3} u_{3}(0, t), \quad t \in(0, T), \\
\left.P_{1}(1, t)+\frac{\rho}{2} u_{1}^{2}(1, t)=P_{2}(0, t)+\frac{\rho}{2} u_{2}^{2}(0, t)=P_{3}(0, t)+\frac{\rho}{2} u_{3}^{2} 0, t\right), \quad t \geqslant 0,
\end{array}\right.
$$

and with the following Dirichlet boundary conditions

$$
u_{1}(0, t)=h_{1}(t), \quad u_{2}(1, t)=h_{2}(t), \quad u_{3}(1, t)=h_{3}(t), \quad t \in(0, T) .
$$

We look for solution to the system (5.9)-(5.11) in the space of functions $\left(A_{i}, u_{i}\right)_{i=1}^{3}$ satisfying

$$
\begin{gathered}
\left(A_{i}\right)_{i=1}^{3} \in H^{1}\left(0, T ; H^{1}(I)\right)^{3}, \quad\left(u_{i}\right)_{i=1}^{3} \in L^{2}\left(0, T ; H^{1}(I)\right)^{3} \cap L^{\infty}\left(0, T ; H^{1}(I)\right)^{3}, \\
\left(A_{i} u_{i}\right)_{i=1}^{3} \in L^{2}\left(0, T ; H^{2}(I)\right)^{3} \cap H^{1}\left(0, T ; L^{2}(I)\right)^{3},
\end{gathered}
$$

equipped with the distance

$$
\begin{aligned}
& d\left(\left(A^{1}, u^{1}\right),\left(A^{2}, u^{2}\right)\right)=\sum_{i=1}^{3}\left(\left\|A_{i}^{1}-A_{i}^{2}\right\|_{H^{1}\left(0, T ; H^{1}(I)\right) \cap L^{\infty}\left(0, T ; H^{1}(I)\right)}\right. \\
& \left.\quad+\left\|u_{i}^{1}-u_{i}^{2}\right\|_{L^{2}\left(0, T ; H^{1}(I)\right) \cap L^{\infty}\left(0, T ; H^{1}(I)\right)}+\left\|A_{i}^{1} u_{i}^{1}-A_{i}^{2} u_{i}^{2}\right\|_{L^{2}\left(0, T ; H^{2}(I)\right) \cap H^{1}\left(0, T ; L^{2}(I)\right) \cap L^{\infty}\left(0, T ; H^{1}(I)\right)}\right),
\end{aligned}
$$

where $A^{j}=\left(A_{1}^{j}, A_{2}^{j}, A_{3}^{j}\right)$ and $u^{j}=\left(u_{1}^{j}, u_{2}^{j}, u_{3}^{j}\right), j=1,2$. As before, let us set

$$
A=\left(A_{i}\right)_{i=1}^{3}, \quad u=\left(u_{i}\right)_{i=1}^{3} .
$$

Let us recall that the constant $\gamma_{A}(0, T)$ is defined in (1.12).

Definition 5.1. We say that a pair $(A, u)$ is a strong solution to system (5.9) - (5.11) over the time interval $[0, T]$ when $(A, u)$ satisfies the regularity assumptions in $(5.12), \gamma_{A}(0, T)>0$, and when $(A, u)$ satisfies $(5.9)$ in the sense of distributions in $I \times(0, T)$ and (5.10)-(5.11) in the sense of traces.

We say that $(A, Q)$ is a maximal strong solution to system (5.9)-(5.10) over the time interval $\left[0, T_{\mathrm{m}}\right)$ when either $T_{\mathrm{m}}=\infty$, or $T_{\mathrm{m}}<\infty$ and, for all $0<T<T_{\mathrm{m}},(A, u)$ is a strong solution to system (5.9)-(5.10) over the time interval $[0, T]$, and when

$$
\lim _{T \rightarrow T_{\mathrm{m}}}\left(d((A, u),(0,0))+\max \left\{\left|A_{i}(x, T)\right|^{-1} \mid 1 \leqslant i \leqslant 3, x \in[0,1]\right\}\right)=\infty .
$$


With the above definition it is quite easy to have an equivalence for the existence of strong solutions between the $(A, Q)$ system $((1.4)-(1.7))$ and the $(A, u)$ system $((5.9)-(5.11))$. More precisely, we have the following result:

Proposition 5.2. Let $(A, Q)$ be a maximal solution to the system (1.4)-(1.7) over $\left[0, T_{m}\right)$ with boundary conditions on the velocity

$$
u_{1}(0, t)=\frac{Q_{1}}{A_{1}}(0, t)=h_{1}(t), \quad u_{2}(1, t)=\frac{Q_{2}}{A_{2}}(1, t)=h_{2}(t), \quad u_{3}(1, t)=\frac{Q_{3}}{A_{3}}(1, t)=h_{3}(t),
$$

( respectively with boundary conditions on the flow rate

$$
\left.Q_{1}(0, t)=h_{1}(t), \quad Q_{2}(1, t)=h_{2}(t), \quad Q_{3}(1, t)=h_{3}(t),\right)
$$

if and only if, $(A, u)$ is a maximal strong solution to the system (5.9)-(5.10), with Dirichlet boundary conditions on the velocity

$$
u_{1}(0, t)=h_{1}(t), \quad u_{2}(1, t)=h_{2}(t), \quad u_{3}(1, t)=h_{3}(t)
$$

( respectively with boundary conditions on the flow rate

$$
\left.u_{1}(0, t)=\frac{h_{1}(t)}{A_{1}(0, t)}, \quad u_{2}(1, t)=\frac{h_{2}(t)}{A_{2}(1, t)}, \quad u_{3}(1, t)=\frac{h_{3}(t)}{A_{3}(1, t)}\right) .
$$

Let us remark that, in Theorem 1.2, we have proved the existence of a unique maximal solution for the $(A, Q)$-system (1.4)-(1.6) with the classical Dirichlet boundary conditions (5.16). Similarly, we can show the existence of a unique maximal solution for the $(A, Q)$-system (1.4)-(1.6) with the nonlinear Dirichlet boundary conditions (5.15). More precisely, we have the following result.

Theorem 5.3. Let us assume that, for $i=1,2,3, A_{i}^{0}>0, A^{0} \in\left[H^{1}(I)\right]^{3}, Q^{0} \in\left[H^{1}(I)\right]^{3}, h_{i} \in H_{\mathrm{loc}}^{3 / 4}([0, \infty))$ satisfying the compatibility conditions

$$
\begin{aligned}
& Q_{1}^{0}(1)=Q_{2}^{0}(0)+Q_{3}^{0}(0), \\
& Q_{1}^{0}(0)=A_{1}^{0}(0) h_{1}(0), \quad Q_{2}^{0}(1)=A_{2}^{0}(1) h_{2}(0), \quad Q_{3}^{0}(1)=A_{3}^{0}(1) h_{3}(0) .
\end{aligned}
$$

Then, the system (1.4)-(1.6) together with the nonlinear Dirichlet conditions (5.15) admits a unique maximal strong solution over $\left[0, T_{\mathrm{m}}\right)$, for some $T_{\mathrm{m}}>0$. Both the solution and the maximal time of existence $T_{\mathrm{m}}$ are unique.

Proof. The proof is similar to that of Theorem 1.2.

We are now in a position to state the existence and uniqueness result for the $(A, u)$ system $(5.9)-(5.10)$. It is an immediate consequence of Theorems 1.2 and 5.3, and of Proposition 5.2.

Theorem 5.4. Let us assume that, for $i=1,2,3, A_{i}^{0}>0, A^{0} \in\left[H^{1}(I)\right]^{3}, u^{0} \in\left[H^{1}(I)\right]^{3}, h_{i} \in H_{\mathrm{loc}}^{3 / 4}([0, \infty))$ satisfying the compatibility conditions

$$
\begin{aligned}
& A_{1}^{0} u_{1}^{0}(1)=A_{2}^{0} u_{2}^{0}(0)+A_{3}^{0} u_{3}^{0}(0), \\
& u_{1}^{0}(0)=h_{1}(0), \quad u_{2}^{0}(1)=h_{2}(0), \quad u_{3}^{0}(1)=h_{3}(0)
\end{aligned}
$$

( respectively the compatibility conditions

$$
\begin{aligned}
& A_{1}^{0} u_{1}^{0}(1)=A_{2}^{0} u_{2}^{0}(0)+A_{3}^{0} u_{3}^{0}(0), \\
& \left.u_{1}^{0}(0)=\frac{h_{1}(0)}{A_{1}^{0}(0)}, \quad u_{2}^{0}(1)=\frac{h_{2}(0)}{A_{2}^{0}(1)}, \quad u_{3}^{0}(1)=\frac{h_{3}(0)}{A_{3}^{0}(1)}\right) .
\end{aligned}
$$

Then, the system (5.9) - (5.10) with the classical Dirichlet boundary conditions (5.17) (respectively the nonlinear Dirichlet boundary conditions (5.18)) admits a unique maximal strong solution over $\left[0, T_{\mathrm{m}}\right)$, for some $T_{\mathrm{m}}>0$. Both the solution and the maximal time of existence $T_{\mathrm{m}}$ are unique. 


\section{Energy estimate For System (1.8)}

We are going to prove an energy identity for the maximal solution to system (1.8) over $\left[0, T_{\mathrm{m}}\right)$. But this energy identity is also valid for solutions which do not satisfy the Dirichlet boundary conditions

$$
Q_{1}(0, t)=h_{1}(t), \quad Q_{2}(1, t)=h_{2}(t), \quad Q_{3}(1, t)=h_{3}(t), \quad t \in(0, T),
$$

of system (1.8). This is why we consider a system corresponding to system 1.8, but in which the Dirichlet boundary conditions are not specified:

For $i \in\{1,2,3\},\left(A_{i}, Q_{i}\right)$ satisfies

$$
\begin{aligned}
& \frac{\partial A_{i}}{\partial t}+\frac{\partial Q_{i}}{\partial x}=0, \quad t \in\left(0, T_{\mathrm{m}}\right), x \in I, \\
& \frac{\rho}{A_{i}} \frac{\partial Q_{i}}{\partial t}+\frac{\rho}{A_{i}}\left(\frac{2 Q_{i}}{A_{i}} \frac{\partial Q_{i}}{\partial x}-\frac{Q_{i}^{2}}{A_{i}^{2}} \frac{\partial A_{i}}{\partial x}\right)+\frac{\beta}{2 A_{i, 0} \sqrt{A_{i}}} \frac{\partial A_{i}}{\partial x}+\frac{\nu}{4 A_{i, 0} A_{i}^{3 / 2}} \frac{\partial A_{i}}{\partial x} \frac{\partial Q_{i}}{\partial x}-\frac{\nu}{2 A_{i, 0} \sqrt{A_{i}}} \frac{\partial^{2} Q_{i}}{\partial x^{2}} \\
& \quad=-k_{f} \rho \frac{Q_{i}}{A_{i}^{2}}, \quad t \in\left(0, T_{\mathrm{m}}\right), x \in I, \\
& Q_{1}(1, t)=Q_{2}(0, t)+Q_{3}(0, t), \quad t \in\left(0, T_{\mathrm{m}}\right), \\
& -\frac{\nu}{2 A_{1,0} \sqrt{A_{1}}} \frac{\partial Q_{1}}{\partial x}(1, t)+\frac{\beta}{A_{1,0}}\left(\sqrt{A_{1}(1, t)}-\sqrt{A_{1,0}}\right)+\frac{1}{2} \rho \frac{Q_{1}^{2}(1, t)}{A_{1}^{2}(1, t)} \\
& \quad=-\frac{\nu}{2 A_{2,0} \sqrt{A_{2}}} \frac{\partial Q_{2}}{\partial x}(0, t)+\frac{\beta}{A_{2,0}}\left(\sqrt{A_{2}(0, t)}-\sqrt{A_{2,0}}\right)+\frac{1}{2} \rho \frac{Q_{2}^{2}(0, t)}{A_{2}^{2}(0, t)} \\
& \quad=-\frac{\nu}{2 A_{3,0} \sqrt{A_{3}}} \frac{\partial Q_{3}}{\partial x}(0, t)+\frac{\beta}{A_{3,0}}\left(\sqrt{A_{3}(0, t)}-\sqrt{A_{3,0}}\right)+\frac{1}{2} \rho \frac{Q_{3}^{2}(0, t)}{A_{3}^{2}(0, t)}, \quad t \in\left(0, T_{\mathrm{m}}\right), \\
& A_{i}(x, 0)=A_{i}^{0}(x), \quad Q_{i}(x, 0)=Q_{i}^{0}(x), \quad x \in I .
\end{aligned}
$$

We set

$$
E(t)=\sum_{i=1}^{3} \frac{1}{2} \int_{0}^{1} \frac{Q_{i}^{2}}{A_{i}}(x, t) \mathrm{d} x+\int_{0}^{1} \frac{2 \beta}{3 \rho A_{i, 0}}\left(\sqrt{A_{i}}-\sqrt{A_{i, 0}}\right)^{3}(x, t) \mathrm{d} x+\int_{0}^{1} \frac{\beta}{\rho \sqrt{A_{i, 0}}}\left(\sqrt{A_{i}}-\sqrt{A_{i, 0}}\right)^{2}(x, t) \mathrm{d} x .
$$

In this section, at first we want to derive an energy estimate for the system (1.8). Let us notice that $E(t) \geqslant 0$ because

$$
\frac{2 \beta}{3 \rho A_{i, 0}}\left(\sqrt{A_{i}}-\sqrt{A_{i, 0}}\right)^{3}+\frac{\beta}{\rho \sqrt{A_{i, 0}}}\left(\sqrt{A_{i}}-\sqrt{A_{i, 0}}\right)^{2}=\frac{2 \beta}{3 \rho A_{i, 0}}\left(\sqrt{A_{i}}-\sqrt{A_{i, 0}}\right)^{2}\left(\sqrt{A_{i}}+\frac{1}{2} \sqrt{A_{i, 0}}\right) .
$$

Lemma 6.1. If $\left(A_{i}, Q_{i}\right)_{i=1}^{3}$ is a maximal solution to system (6.1) over $\left[0, T_{\mathrm{m}}\right)$, then we have

$$
\begin{aligned}
E(t)+\int_{0}^{t} \int_{0}^{1} \frac{\nu}{2 A_{i, 0} \rho \sqrt{A_{i}}}\left(\frac{\partial A_{i}}{\partial t}\right)^{2}(x, \tau) \mathrm{d} x \mathrm{~d} \tau+k_{f} \int_{0}^{t} \int_{0}^{1} \frac{Q_{i}^{2}}{A_{i}^{2}} \mathrm{~d} x \mathrm{~d} \tau \\
+\frac{1}{\rho} \int_{0}^{t}\left(Q_{2}\left(P_{2}(1, \tau)-P_{\text {ext }}+\frac{1}{2} \rho u_{2}^{2}(1, \tau)\right) \mathrm{d} \tau+\frac{1}{\rho} \int_{0}^{t}\left(Q_{3}\left(P_{3}(1, \tau)-P_{\text {ext }}+\frac{1}{2} \rho u_{3}^{2}(1, \tau)\right) \mathrm{d} \tau\right.\right. \\
-\frac{1}{\rho} \int_{0}^{t}\left(Q_{1}\left(P_{1}(0, \tau)-P_{\text {ext }}+\frac{1}{2} \rho u_{1}^{2}(0, \tau)\right) \mathrm{d} \tau=E(0),\right.
\end{aligned}
$$

for all $t \in\left[0, T_{\mathrm{m}}\right)$, where, for $i=1,2,3, P_{i}$ is defined by $(1.4)_{4}$.

Proof. We multiply equation $(6.1)_{3}$ by $u_{i}:=\frac{Q_{i}}{A_{i}}$, we replace $Q_{i}$ by $A_{i} u_{i}$, and we integrate over $I$. We end up with four terms. Let us analyse the four terms separately. 
First term.

$$
\int_{0}^{1} \frac{\partial}{\partial t}\left(A_{i} u_{i}\right) \cdot u=\int_{0}^{1}\left(\frac{\partial A_{i}}{\partial t} \cdot u_{i}^{2}+\frac{A_{i}}{2} \frac{\partial}{\partial t}\left(u_{i}^{2}\right)\right)=\frac{1}{2} \int_{0}^{1} \frac{\partial A_{i}}{\partial t} \cdot u_{i}^{2}+\frac{1}{2}\left(\int_{0}^{1} \frac{\partial A_{i}}{\partial t} \cdot u_{i}^{2}+A \cdot \frac{\partial}{\partial t}\left(u_{i}^{2}\right)\right) .
$$

Thus,

$$
\int_{0}^{1} \frac{\partial}{\partial t}\left(A_{i} u_{i}\right) \cdot u_{i}=\frac{1}{2} \int_{0}^{1} \frac{\partial A_{i}}{\partial t} \cdot u_{i}^{2}+\frac{1}{2} \int_{0}^{1} \frac{\partial}{\partial t}\left(A_{i} u_{i}^{2}\right)
$$

Second term.

$$
\int_{0}^{1} \frac{\partial}{\partial x}\left(A_{i} u_{i}^{2}\right) \cdot u_{i}=\int_{0}^{1}\left(\frac{\partial}{\partial x}\left(A_{i} u_{i}\right) \cdot u_{i}^{2}+A u^{2} \cdot \frac{\partial u}{\partial x}\right)=\int_{0}^{1}\left(\frac{1}{2} \frac{\partial}{\partial x}(A u) \cdot u^{2}+\frac{1}{2} \frac{\partial A_{i}}{\partial x} u_{i}^{3}+\frac{3}{2} A_{i} u_{i}^{2} \cdot \frac{\partial u_{i}}{\partial x}\right)
$$

It can be written as

$$
\int_{0}^{1} \frac{\partial}{\partial x}\left(A_{i} u_{i}^{2}\right) \cdot u=\frac{1}{2} \int_{0}^{1}\left(\frac{\partial Q_{i}}{\partial x} \cdot u_{i}^{2}+\frac{\partial}{\partial x}\left(A_{i} u_{i}^{3}\right)\right)=-\frac{1}{2} \int_{0}^{1} \frac{\partial A_{i}}{\partial t} \cdot u_{i}^{2}+\frac{1}{2}\left(Q_{i}(1, t) u_{i}^{2}(1, t)-Q_{i}(0, t) u_{i}^{2}(0, t)\right) .
$$

\section{Third term.}

$$
\begin{array}{r}
\frac{1}{\rho} \int_{0}^{1} A_{i} \frac{\partial P_{i}}{\partial x} u_{i}=\frac{1}{\rho}\left[-\int_{0}^{1} \frac{\partial Q_{i}}{\partial x}\left(P_{i}-P_{\text {ext }}\right)+Q_{i}(1, t)\left(P_{i}(1, t)-P_{\text {ext }}\right)-Q_{i}(0, t)\left(P_{i}(0, t)-P_{\text {ext }}\right)\right] \\
=\frac{\nu}{2 A_{i, 0} \rho} \int_{0}^{1} \frac{1}{\sqrt{A_{i}}}\left(\frac{\partial A_{i}}{\partial t}\right)^{2}+\frac{\beta}{A_{i, 0} \rho} \int_{0}^{1} \frac{\partial A_{i}}{\partial t}\left(\sqrt{A_{i}}-\sqrt{A_{i, 0}}\right) \\
+\frac{1}{\rho} Q_{i}(1, t)\left(P_{i}(1, t)-P_{\text {ext }}\right)-\frac{1}{\rho} Q_{i}(0, t)\left(P_{i}(0, t)-P_{\text {ext }}\right)
\end{array}
$$

We have

$$
\begin{array}{r}
\int_{0}^{1} \frac{\partial A_{i}}{\partial t}\left(\sqrt{A_{i}}-\sqrt{A_{i, 0}}\right)=\int_{0}^{1} \frac{\partial \sqrt{A_{i}}}{\partial t}\left(\sqrt{A_{i}}-\sqrt{A_{i, 0}}\right) 2 \sqrt{A_{i}}=\int_{0}^{1} \frac{\partial\left(\sqrt{A_{i}}-\sqrt{A_{i, 0}}\right)}{\partial t}\left(\sqrt{A_{i}}-\sqrt{A_{i, 0}}\right) 2 \sqrt{A_{i}} \\
=\frac{2}{3} \int_{0}^{1} \frac{\partial}{\partial t}\left(\sqrt{A_{i}}-\sqrt{A_{i, 0}}\right)^{3}+\int_{0}^{1} \sqrt{A_{i, 0}} \frac{\partial}{\partial t}\left(\sqrt{A_{i}}-\sqrt{A_{i, 0}}\right)^{2}
\end{array}
$$

This helps us to rewrite the third term.

Fourth term.

$$
K_{f} \int_{0}^{1} u_{i}^{2} \mathrm{~d} x
$$


Thus, by adding (6.4), (6.5), (6.6) and (6.8), we obtain

$$
\begin{gathered}
\sum_{i=1}^{3}\left(\frac{1}{2} \frac{d}{d t} \int_{0}^{1} A_{i} u_{i}^{2} d x+\int_{0}^{1} \frac{\nu}{2 A_{i, 0} \rho \sqrt{A_{i}}}\left(\frac{\partial A_{i}}{\partial t}\right)^{2} d x+\frac{\beta}{\rho \sqrt{A_{i, 0}}} \frac{d}{d t} \int_{0}^{1}\left(\sqrt{A_{i}}-\sqrt{A_{i, 0}}\right)^{2} d x\right. \\
\left.+\frac{2 \beta}{3 \rho A_{i, 0}} \frac{d}{d t} \int_{0}^{1}\left(\sqrt{A_{i}}-\sqrt{A_{i, 0}}\right)^{3} d x+k_{f} \int_{0}^{1} u_{i}^{2} d x\right) \\
+\frac{1}{\rho} \sum_{i=1}^{3}\left(Q_{i}\left(P_{i}-P_{\text {ext }}+\frac{1}{2} u_{i}^{2}\right)(1, t)-Q_{i}\left(P_{i}-P_{\text {ext }}+\frac{1}{2} u_{i}^{2}\right)(0, t)\right)=0 .
\end{gathered}
$$

As $Q_{1}(1, t)=Q_{2}(0, t)+Q_{3}(0, t)$ and

we have

$$
\left(P_{1}-P_{\text {ext }}+\frac{1}{2} u_{1}^{2}\right)(1, t)=\left(P_{2}-P_{\text {ext }}+\frac{1}{2} u_{2}^{2}\right)(0, t)=\left(P_{3}-P_{\text {ext }}+\frac{1}{2} u_{3}^{2}\right)(0, t),
$$

$$
\begin{aligned}
& \sum_{i=1}^{3}\left(Q_{i}\left(P_{i}-P_{\mathrm{ext}}+\frac{1}{2} u_{i}^{2}\right)(1, t)-Q_{i}\left(P_{i}-P_{\mathrm{ext}}+\frac{1}{2} u_{i}^{2}\right)(0, t)\right) \\
& =Q_{2}\left(P_{2}-P_{\mathrm{ext}}+\frac{1}{2} \rho u_{2}^{2}\right)(1, t)+Q_{3}\left(P_{3}-P_{\mathrm{ext}}+\frac{1}{2} \rho u_{3}^{2}\right)(1, t)-Q_{1}\left(P_{1}-P_{\mathrm{ext}}+\frac{1}{2} \rho u_{1}^{2}\right)(0, t) .
\end{aligned}
$$

The proof is complete.

If, in the energy identity (6.3), we substitute $Q_{1}(0, t), Q_{2}(1, t)$, and $Q_{3}(2, t)$ by $h_{1}(t), h_{2}(t)$ and $h_{3}(t)$ respectively, we do not obtain a stability estimate unless $h_{1}(t)=h_{2}(t)=h_{3}(t)=0$. We are going to show that we can obtain a stability estimate if we replace the Dirichlet boundary conditions

$$
Q_{1}(0, t)=h_{1}(t), \quad Q_{2}(1, t)=h_{2}(t), \quad \text { and } \quad Q_{3}(1, t)=h_{3}(t),
$$

by the following nonlinear Robin boundary conditions

$$
\begin{aligned}
& -\varepsilon \frac{\nu}{2 A_{1,0} \sqrt{A_{1}^{0}}} \frac{\partial Q_{1}}{\partial x}(0, t)+Q_{1}(0, t)=\varepsilon H_{1}\left(Q_{1}, A_{1}\right)+h_{1}(t), \quad t \in\left(0, T_{\mathrm{m}}\right), \\
& \text { and for } i=1,2
\end{aligned}
$$

$$
\varepsilon \frac{\nu}{2 A_{i, 0} \sqrt{A_{i}^{0}}} \frac{\partial Q_{i}}{\partial x}(1, t)+Q_{i}(1, t)=\varepsilon H_{i}\left(Q_{i}, A_{i}\right)+h_{i}(t), \quad t \in\left(0, T_{\mathrm{m}}\right)
$$

with

$$
H_{1}\left(Q_{1}, A_{1}\right)=\left.\left[-\frac{\beta}{A_{1,0}}\left(\sqrt{A_{1}}-\sqrt{A_{1,0}}\right)+\frac{\nu}{2 A_{1,0}} \frac{\partial Q_{1}}{\partial x}\left(\frac{1}{\sqrt{A_{1}}}-\frac{1}{\sqrt{A_{1}^{0}}}\right)-\frac{1}{2} \rho \frac{Q_{1}^{2}}{A_{1}^{2}}\right]\right|_{x=0}
$$

and for $i=1,2$,

$$
H_{i}\left(Q_{i}, A_{i}\right)=\left.\left[\frac{\beta}{A_{i, 0}}\left(\sqrt{A_{i}}-\sqrt{A_{i, 0}}\right)-\frac{\nu}{2 A_{i, 0}} \frac{\partial Q_{i}}{\partial x}\left(\frac{1}{\sqrt{A_{i}}}-\frac{1}{\sqrt{A_{i}^{0}}}\right)+\frac{1}{2} \rho \frac{Q_{i}^{2}}{A_{i}^{2}}\right]\right|_{x=1} .
$$

For the network represented in Figure 1, we consider the system (6.1) with the nonlinear Robin boundary conditions (6.11)-(6.12).

The notions of strong solution to system (6.1)-(6.11)-(6.12) over the time interval $[0, T]$, and of maximal strong solution over the time interval $\left[0, T_{\mathrm{m}}\right)$, are similar to those in Definition 1.1.

Theorem 6.2. Let us assume that, for $i=1,2,3, A_{i}^{0}>0, A^{0} \in\left[H^{1}(I)\right]^{3}, Q^{0} \in\left[H^{1}(I)\right]^{3}, h_{i} \in H_{\mathrm{loc}}^{3 / 4}([0, \infty))$ and

$$
Q_{1}^{0}(1)=Q_{2}^{0}(0)+Q_{3}^{0}(0)
$$

Then, the system (6.1)-(6.11)-(6.12) admits a unique maximal strong solution over $\left[0, T_{\mathrm{m}}\right)$, for some $T_{\mathrm{m}}>0$. Both the solution and the maximal time of existence $T_{\mathrm{m}}$ are unique.

Proof. The proof is similar to that of Theorem 1.2. 
Proposition 6.3. The maximal solution $\left(A_{i}, Q_{i}\right)_{i=1}^{3}$ to system (6.1)-(6.11)-(6.12) over $\left[0, T_{\mathrm{m}}\right)$, whose existence is stated in Theorem 6.2, satisfies the following stability estimate

$$
\begin{aligned}
& E(t)+\sum_{i=1}^{3} \int_{0}^{t} \int_{0}^{1} \frac{\nu}{2 A_{i, 0} \rho \sqrt{A_{i}}}\left(\frac{\partial A_{i}}{\partial t}\right)^{2}(x, \tau) \mathrm{d} x \mathrm{~d} \tau+k_{f} \sum_{i=1}^{3} \int_{0}^{t} \int_{0}^{1} \frac{Q_{i}^{2}}{A_{i}^{2}} \mathrm{~d} x \mathrm{~d} \tau \\
& +\frac{1}{2 \rho \varepsilon} \int_{0}^{t} Q_{2}^{2}(1, \tau) \mathrm{d} \tau+\frac{1}{2 \rho \varepsilon} \int_{0}^{t} Q_{3}^{2}(1, \tau) \mathrm{d} \tau+\frac{1}{2 \rho \varepsilon} \int_{0}^{t} Q_{1}^{2}(0, \tau) \mathrm{d} \tau \\
& \leqslant E(0)+\frac{1}{2 \rho \varepsilon} \int_{0}^{t} h_{2}^{2}(1, \tau) \mathrm{d} \tau+\frac{1}{2 \rho \varepsilon} \int_{0}^{t} h_{3}^{2}(1, \tau) \mathrm{d} \tau+\frac{1}{2 \rho \varepsilon} \int_{0}^{t} h_{1}^{2}(0, \tau) \mathrm{d} \tau, \quad \text { for all } t \in\left[0, T_{m}\right) .
\end{aligned}
$$

Proof. Due to Lemma 6.1, to prove the proposition, it is sufficient to estimate the following boundary terms

$$
\begin{aligned}
& \int_{0}^{t}\left(Q_{2}(1, \tau)\left(P_{2}(1, \tau)-P_{\text {ext }}+\frac{1}{2} \rho u_{2}^{2}(1, \tau)\right) \mathrm{d} \tau, \quad \int_{0}^{t}\left(Q_{3}(1, \tau)\left(P_{3}(1, \tau)-P_{\text {ext }}+\frac{1}{2} \rho u_{3}^{2}(1, \tau)\right) \mathrm{d} \tau\right.\right. \\
& \text { and } \int_{0}^{t}\left(Q_{1}(0, \tau)\left(P_{1}(0, \tau)-P_{\text {ext }}+\frac{1}{2} \rho u_{1}^{2}(0, \tau)\right) \mathrm{d} \tau .\right.
\end{aligned}
$$

Let us only estimate the last term, the two others can be estimated similarly. The Robin boundary condition satisfied by $Q_{1}$ can be written in the form

$$
\begin{aligned}
& -Q_{1}(0, \tau)+h_{1}(t)=\varepsilon\left(\frac{\beta}{A_{1,0}}\left(\sqrt{A_{1}}-\sqrt{A_{1,0}}\right)-\frac{\nu}{2 A_{1,0} \sqrt{A_{1}}} \frac{\partial Q_{1}}{\partial x}+\frac{1}{2} \rho \frac{Q_{1}^{2}}{A_{1}^{2}}(0, \tau)\right) \\
& =\varepsilon\left(\frac{\beta}{A_{1,0}}\left(\sqrt{A_{1}}-\sqrt{A_{1,0}}\right)+\frac{\nu}{2 A_{1,0} \sqrt{A_{1}}} \frac{\partial A_{1}}{\partial t}+\frac{1}{2} \rho \frac{Q_{1}^{2}}{A_{1}^{2}}(0, \tau)\right) \\
& =\varepsilon\left(P_{1}(0, \tau)-P_{\text {ext }}+\frac{1}{2} \rho u_{1}^{2}(0, \tau)\right) .
\end{aligned}
$$

Thus, we have

$$
\varepsilon\left(P_{1}(0, \tau)-P_{\mathrm{ext}}+\frac{1}{2} \rho u_{1}^{2}(0, \tau)\right)=-Q_{1}(0, \tau)+h_{1}(t) .
$$

and

$$
\begin{aligned}
& -\int_{0}^{t} Q_{1}(0, \tau)\left(P_{1}(0, \tau)-P_{\text {ext }}+\frac{1}{2} \rho u_{1}^{2}(0, \tau)\right) \mathrm{d} \tau \\
& =\frac{1}{\varepsilon} \int_{0}^{t} Q_{1}(0, \tau)\left(Q_{1}(0, \tau)-h_{1}(\tau)\right) \mathrm{d} \tau \geqslant \frac{1}{2 \varepsilon} \int_{0}^{t} Q_{1}^{2}(0, \tau) \mathrm{d} \tau-\frac{1}{2 \varepsilon} \int_{0}^{t} h_{1}^{2}(\tau) \mathrm{d} \tau .
\end{aligned}
$$

The nonlinear Robin boundary conditions at $x=1$ satisfied by $Q_{i}$ with $i=2,3$, correspond to

$$
P_{i}(1, \tau)-P_{\mathrm{ext}}+\frac{1}{2} \rho u_{i}^{2}(0, \tau)=Q_{i}(1, \tau)-h_{i}(t)
$$

Thus, for $i=2,3$, we have

$$
\int_{0}^{t}\left(Q_{2}(1, \tau)\left(P_{2}(1, \tau)-P_{\text {ext }}+\frac{1}{2} \rho u_{2}^{2}(1, \tau)\right) \mathrm{d} \tau \geqslant \frac{1}{2 \varepsilon} \int_{0}^{t} Q_{i}^{2}(0, \tau) \mathrm{d} \tau-\frac{1}{2 \varepsilon} \int_{0}^{t} h_{i}^{2}(\tau) \mathrm{d} \tau,\right.
$$

and the proof is complete. 


\section{A GENERAL NETWORK}

We now consider a network constituted of $N_{s}$ segments of length 1 , numbered from $i=1$ to $i=N_{s}$ and parametrized by $x \in I=(0,1)$. The origins and extremities of the segments, corresponding to the points where $x=0$ and $x=1$ respectively, are the nodes of the network. The origins of segments are either inlet points or branching points, while the extremities are either outlet points or branching points. The set of branching points is $\left\{B_{\ell} \mid 1 \leqslant \ell \leqslant N_{b}\right\}$. At any branching point $B_{\ell}$, the subsets of indices $J_{0}^{\ell} \subset\left\{1, \cdots, N_{s}\right\}$ and $J_{1}^{\ell} \subset\left\{1, \cdots, N_{s}\right\}$, corresponding to segments connected to $B_{\ell}$ by their origin and their extremity respectively, are nonempty. The subsets of indices $J_{\text {in }} \subset\left\{1, \cdots, N_{s}\right\}$ and $J_{\text {out }} \subset\left\{1, \cdots, N_{s}\right\}$ are nonempty, and they correspond to origins of segments which are inlet points and extremities of segments which are outlet points respectively. The boundary conditions at a branching point $B_{\ell}$ are

$$
\begin{aligned}
& \sum_{i \in J_{0}^{\ell}} Q_{i}(0, t)=\sum_{i \in J_{1}^{\ell}} Q_{i}(1, t), \\
& P_{j_{0}^{\ell}}(0, t)+\frac{1}{2} \rho u_{j_{0}^{\ell}}^{2}(0, t)=P_{j}(0, t)+\frac{1}{2} \rho u_{j}^{2}(0, t) \quad \text { for all } j \in J_{0}^{\ell}, \\
& P_{j_{0}^{\ell}}(0, t)+\frac{1}{2} \rho u_{j_{0}^{\ell}}^{2}(0, t)=P_{j}(1, t)+\frac{1}{2} \rho u_{j}^{2}(1, t) \quad \text { for all } j \in J_{1}^{\ell},
\end{aligned}
$$

where $j_{0}^{\ell}=\min J_{0}^{\ell}$.

For this type of network, we consider the nonlinear system

$$
\begin{aligned}
& \text { For } i \in\left\{1, \cdots, N_{s}\right\},\left(A_{i}, Q_{i}, u_{i}\right), \text { with } u_{i}=\frac{Q_{i}}{A_{i}}, \text { satisfies } \\
& \frac{\partial A_{i}}{\partial t}+\frac{\partial Q_{i}}{\partial x}=0, \quad t \in(0, T), x \in I, \\
& \frac{\partial Q_{i}}{\partial t}+\frac{\partial}{\partial x}\left(\frac{Q_{i}^{2}}{A_{i}}\right)+\frac{A_{i}}{\rho} \frac{\partial P_{i}}{\partial x}=-k_{f} \frac{Q_{i}}{A_{i}}, \quad t \in(0, T), x \in I \\
& P_{i}=P_{\text {ext }}+\frac{\beta}{A_{i, 0}}\left(\sqrt{A_{i}}-\sqrt{A_{i, 0}}\right)+\frac{\nu}{A_{i, 0}} \frac{\partial}{\partial t}\left(\sqrt{A_{i}}\right), \\
& A_{i}(x, 0)=A_{i}^{0}, \quad Q_{i}(x, 0)=Q_{i}^{0}(x), \quad x \in I,
\end{aligned}
$$

with the following nonlinear boundary conditions at the branching points

$$
\begin{aligned}
& \sum_{i \in J_{0}^{\ell}} Q_{i}(0, t)=\sum_{i \in J_{1}^{\ell}} Q_{i}(1, t), \text { for all } 1 \leqslant \ell \leqslant N_{b} \\
& P_{j_{0}^{\ell}}(0, t)+\frac{1}{2} \rho u_{j_{0}^{\ell}}^{2}(0, t)=P_{j}(0, t)+\frac{1}{2} \rho u_{j}^{2}(0, t) \quad \text { for all } 1 \leqslant \ell \leqslant N_{b}, \text { and all } j \in J_{0}^{\ell}, \\
& P_{j_{0}^{\ell}}(0, t)+\frac{1}{2} \rho u_{j_{0}^{\ell}}^{2}(0, t)=P_{j}(1, t)+\frac{1}{2} \rho u_{j}^{2}(1, t) \quad \text { for all } 1 \leqslant \ell \leqslant N_{b}, \text { and all } j \in J_{1}^{\ell},
\end{aligned}
$$

with either the following Dirichlet boundary conditions

$$
\begin{aligned}
& Q_{i}(0, t)=h_{i}(t), i \in J_{\text {in }}, t \in(0, T), \\
& Q_{i}(1, t)=h_{i}(t), i \in J_{\text {out }}, t \geqslant 0,
\end{aligned}
$$

or the following nonlinear Robin boundary conditions

$$
\begin{aligned}
& -\varepsilon \frac{\nu}{2 A_{i, 0} \sqrt{A_{i}^{0}}} \frac{\partial Q_{i}}{\partial x}(0, t)+Q_{i}(0, t)=\varepsilon H_{i}^{\text {in }}\left(Q_{1}, A_{1}\right)+h_{1}(t), i \in J_{\text {in }}, t \in(0, T), \\
& \varepsilon \frac{\nu}{2 A_{i, 0} \sqrt{A_{i}^{0}}} \frac{\partial Q_{i}}{\partial x}(1, t)+Q_{i}(1, t)=\varepsilon H_{i}^{\text {out }}\left(Q_{i}, A_{i}\right)+h_{i}(t), i \in J_{\text {out }}, t \in(0, T),
\end{aligned}
$$


where

$$
H_{i}^{\mathrm{in}}\left(A_{i}, Q_{i}\right)=\left.\left[-\frac{\beta}{A_{i, 0}}\left(\sqrt{A_{i}}-\sqrt{A_{i, 0}}\right)+\frac{\nu}{2 A_{i, 0}} \frac{\partial Q_{i}}{\partial x}\left(\frac{1}{\sqrt{A_{i}}}-\frac{1}{\sqrt{A_{i}^{0}}}\right)-\frac{1}{2} \rho \frac{Q_{i}^{2}}{A_{i}^{2}}\right]\right|_{x=0}, \quad i \in J_{\mathrm{in}},
$$

and

$$
H_{i}^{\text {out }}\left(A_{i}, Q_{i}\right)=\left.\left[\frac{\beta}{A_{i, 0}}\left(\sqrt{A_{i}}-\sqrt{A_{i, 0}}\right)-\frac{\nu}{2 A_{i, 0}} \frac{\partial Q_{i}}{\partial x}\left(\frac{1}{\sqrt{A_{i}}}-\frac{1}{\sqrt{A_{i}^{0}}}\right)+\frac{1}{2} \rho \frac{Q_{i}^{2}}{A_{i}^{2}}\right]\right|_{x=1}, \quad i \in J_{\text {out }} .
$$

We look for solutions to system (7.2)-(7.3), or system (7.2)-(7.4), in the space

$$
\begin{aligned}
& \mathcal{E}_{T}=\left\{\left(A_{i}, Q_{i}\right)_{i=1}^{N_{s}} \mid A_{i} \in H^{1}\left(0, T ; H^{1}(0,1)\right) \cap L^{\infty}\left(0, T ; H^{1}(0,1)\right),\right. \\
& \left.Q_{i} \in L^{2}\left(0, T ; H^{2}(0,1)\right) \cap H^{1}\left(0, T ; L^{2}(0,1)\right) \cap L^{\infty}\left(0, T ; H^{1}(0,1)\right), A_{i}(\cdot, 0)=A_{i}^{0} \text { in }(0,1)\right\},
\end{aligned}
$$

equipped with the norm

$$
\begin{gathered}
\left\|\left(A_{i}, Q_{i}\right)_{i=1}^{N_{s}}\right\| \|_{\mathcal{E}_{T}}=\sum_{i=1}^{N_{s}}\left(\left\|A_{i}\right\|_{H^{1}\left(0, T ; H^{1}(0,1)\right)}+\left\|A_{i}\right\|_{L^{\infty}\left(0, T ; H^{1}(0,1)\right)}+\left\|Q_{i}\right\|_{L^{2}\left(0, T ; H^{2}(0,1)\right)}\right. \\
\left.+\left\|Q_{i}\right\|_{H^{1}\left(0, T ; L^{2}(0,1)\right)}+\left\|Q_{i}\right\|_{L^{\infty}\left(0, T ; H^{1}(0,1)\right)}\right) .
\end{gathered}
$$

Definition 7.1. We say that $\left(A_{i}, Q_{i}\right)_{i=1}^{N_{s}}$ is a strong solution to system (7.2)-(7.3) (or system (7.2)-(7.4)), over the time interval $[0, T]$, when $\left(A_{i}, Q_{i}\right)_{i=1}^{N_{s}} \in \mathcal{E}_{T}$,

$$
A_{i}(x, t)>0 \text { for all }(x, t) \in[0,1] \times[0, T],
$$

and $\left(A_{i}, Q_{i}\right)_{i=1}^{N_{s}}$ satisfies the equations $(7.2)_{2-3}$ in the sense of distributions and the boundary and initial conditions in the sense of traces.

We say that $\left(A_{i}, Q_{i}\right)_{i=1}^{N_{s}}$ is a maximal strong solution to system (7.2)-(7.3) (or system (7.2)-(7.4)), over the time interval $\left[0, T_{\mathrm{m}}\right)$, when either $T_{\mathrm{m}}=\infty$, or $T_{\mathrm{m}}<\infty$ and, for all $0<T<T_{\mathrm{m}},\left(A_{i}, Q_{i}\right)_{i=1}^{N_{s}}$ is a strong solution to system (7.2) over the time interval $[0, T]$, and when

$$
\lim _{T \rightarrow T_{\mathrm{m}}}\left(\left\|\mid\left(A_{i}, Q_{i}\right)_{i=1}^{N_{s}}\right\|_{\mathcal{E}_{T}}+\max \left\{\left|A_{i}(x, T)\right|^{-1} \mid 1 \leqslant i \leqslant N_{s}, x \in[0,1]\right\}\right)=\infty .
$$

Theorem 7.2. Let $A_{i, 0}>0$ denote the sectional area of the ith vessel at equilibrium state, and let $Q_{i, 0}$ belong to $H^{1}(I)$, and let assume that

$$
\sum_{i \in J_{0}^{\ell}} Q_{i}(0, t)=\sum_{i \in J_{1}^{\ell}} Q_{i}(0, t) \quad \text { for all } \ell \in\left\{1, \cdots, N_{b}\right\} .
$$

Then, the system (7.2)-(7.3) (or system (7.2)-(7.4)) admits a unique maximal solution over a time interval $\left[0, T_{\mathrm{m}}\right)$.

Proof. The proof is similar to those of Theorems 1.2 and 6.2.

We set

$$
E(t)=\sum_{i=1}^{N_{s}} \frac{1}{2} \int_{0}^{1} \frac{Q_{i}^{2}}{A_{i}}(x, t) \mathrm{d} x+\int_{0}^{1} \frac{2 \beta}{3 \rho A_{i, 0}}\left(\sqrt{A_{i}}-\sqrt{A_{i, 0}}\right)^{3}(x, t) \mathrm{d} x+\int_{0}^{1} \frac{\beta}{\rho \sqrt{A_{i, 0}}}\left(\sqrt{A_{i}}-\sqrt{A_{i, 0}}\right)^{2}(x, t) \mathrm{d} x .
$$

Proposition 7.3. The maximal solution $\left(A_{i}, Q_{i}, P_{i}\right)_{i=1}^{N_{s}}$ to system $(7.2)-(7.4)$, over $\left[0, T_{\mathrm{m}}\right)$, whose existence is stated in Theorem 7.2, satisfies the following stability estimate

$$
\begin{aligned}
& E(t)+\sum_{i=1}^{N_{s}} \int_{0}^{t} \int_{0}^{1} \frac{\nu}{2 A_{i, 0} \rho \sqrt{A_{i}}}\left(\frac{\partial A_{i}}{\partial t}\right)^{2}(x, \tau) \mathrm{d} x \mathrm{~d} \tau+k_{f} \sum_{i=1}^{N_{s}} \int_{0}^{t} \int_{0}^{1} \frac{Q_{i}^{2}}{A_{i}^{2}} \mathrm{~d} x \mathrm{~d} \tau \\
& \leqslant E(0)+\frac{1}{2 \rho \varepsilon} \sum_{i \in J_{\text {in }} \cup J_{\text {out }}} \int_{0}^{t} h_{i}^{2}(\tau) \mathrm{d} \tau, \quad \text { for all } \in\left[0, T_{m}\right) .
\end{aligned}
$$


Proof. The proof is similar to that of Proposition 6.3.

\section{REFERENCES}

[1] R. L. Armentano, J. G. Barra, J. Levenson, A. Simon, and R. H. Pichel, Arterial wall mechanics in conscious dogs. assessment of viscous, inertial, and elastic moduli to characterize aortic wall behavior, Circ. Res., 76 (1995), pp. 468-478.

[2] A. Bensoussan, G. Da Prato, M. C. Delfour, and S. K. Mitter, Representation and control of infinite dimensional systems, Systems \& Control: Foundations \& Applications, Birkhäuser Boston, Inc., Boston, MA, second ed., 2007.

[3] P. J. Blanco, S. M. Watanabe, E. A. Dari, M. A. R. F. Passos, and R. A. Feijóo, Blood flow distribution in an anatomically detailed arterial network model: criteria and algorithms, Biomechanics and Modeling in Mechanobiology, 13 (2014), pp. 1303-1330.

[4] P. J. Blanco, S. M. Watanabe, M. A. R. F. Passos, P. A. Lemos, and R. A. Feijóo, An anatomically detailed arterial network model for one-dimensional computational hemodynamics, IEEE Transactions on Biomedical Engineering, 62 (2015), pp. $736-753$.

[5] S. Čanić, C. J. Hartley, D. Rosenstrauch, J. Tambača, G. Guidoboni, and A. Mikelić, Blood flow in compliant arteries: An effective viscoelastic reduced model, numerics, and experimental validation, Annals of Biomedical Engineering, 34 (2006), pp. $575-592$.

[6] S. CANIĆ AND E. H. KIM, Mathematical analysis of the quasilinear effects in a hyperbolic model blood flow through compliant axi-symmetric vessels, Mathematical Methods in the Applied Sciences, 26 (2003), pp. 1161-1186.

[7] K. DeVault, P. A. Gremaud, V. Novak, M. S. Olufsen, G. Vernières, and P. Zhao, Blood flow in the circle of Willis: modeling and calibration, Multiscale Model. Simul., 7 (2008), pp. 888-909.

[8] L. C. Evans, Partial differential equations, vol. 19 of Graduate Studies in Mathematics, American Mathematical Society, Providence, RI, second ed., 2010.

[9] M. A. Fernández, V. Milišić, And A. Quarteroni, Analysis of a geometrical multiscale blood flow model based on the coupling of ODEs and hyperbolic PDEs, Multiscale Model. Simul., 4 (2005), pp. 215-236.

[10] L. Formaggia, J. F. Gerbeau, F. Nobile, and A. Quarteroni, On the coupling of $3 D$ and $1 D$ Navier-Stokes equations for flow problems in compliant vessels, Comput. Methods Appl. Mech. Engrg., 191 (2001), pp. 561-582.

11] P. Grisvard, Caractérisation de quelques espaces d'interpolation, Arch. Rational Mech. Anal., 25 (1967), pp. 40-63.

[12] G. Grubb And V. A. Solonnikov, Boundary value problems for the nonstationary Navier-Stokes equations treated by pseudodifferential methods, Math. Scand., 69 (1991), pp. 217-290 (1992).

[13] O. A. LadyžEnskaja, V. A. Solonnikov, and N. N. Ural'CeVA, Linear and quasilinear equations of parabolic type, Translated from the Russian by S. Smith. Translations of Mathematical Monographs, Vol. 23, American Mathematical Society, Providence, R.I., 1968.

[14] R. Lal, B. Mohammadi, and F. Nicoud, Data assimilation for identification of cardiovascular network characteristics, Int. J. Numer. Methods Biomed. Eng., 33 (2017), pp. e2824, 17.

[15] J.-L. Lions and E. Magenes, Non-homogeneous boundary value problems and applications. Vol. II, Springer-Verlag, New York-Heidelberg, 1972. Translated from the French by P. Kenneth, Die Grundlehren der mathematischen Wissenschaften, Band 182.

[16] D. Maity, J.-P. RAYmond, AND A. Roy, Maximal-in-time existence and uniqueness of strong solution of a $3 d$ fluid-structure interaction model, https://hal.archives-ouvertes.fr/hal-02912001, (2020).

[17] A. C. I. Malossi, P. J. Blanco, and S. Deparis, A two-level time step technique for the partitioned solution of onedimensional arterial networks, Comput. Methods Appl. Mech. Engrg., 237/240 (2012), pp. 212-226.

[18] G. I. Montecinos, L. O. Müller, AND E. F. Toro, Hyperbolic reformulation of a $1 D$ viscoelastic blood flow model and ADER finite volume schemes, J. Comput. Phys., 266 (2014), pp. 101-123.

[19] L. O. Müller, G. Leugering, And P. J. Blanco, Consistent treatment of viscoelastic effects at junctions in one-dimensional blood flow models, J. Comput. Phys., 314 (2016), pp. 167-193.

[20] R. Raghu And C. A. TAYlor, Verification of a one-dimensional finite element method for modeling blood flow in the cardiovascular system incorporating a viscoelastic wall model, Finite Elem. Anal. Des., 47 (2011), pp. 586-592.

[21] P. Reymond, F. Merenda, F. Perrin, D. Rüfenacht, and N. Stergiopulos, Validation of a one-dimensional model of the systemic arterial tree, Am. J. Physiol. Heart Circ. Physiol., 297 (2009), pp. H208-H222.

[22] B. N. Steele, D. Valdez-Jasso, M. A. Haider, and M. S. Olufsen, Predicting arterial flow and pressure dynamics using a $1 D$ fluid dynamics model with a viscoelastic wall, SIAM J. Appl. Math., 71 (2011), pp. 1123-1143.

[23] M. Strocchi, C. Contarino, Q. Zhang, R. Bonmassari, and E. F. Toro, A global mathematical model for the simulation of stenoses and bypass placement in the human arterial system, Appl. Math. Comput., 300 (2017), pp. 21-39.

[24] X. Wang, J.-M. Fullana, And P.-Y. Lagrée, Verification and comparaison of four numerical schemes for a $1 d$ viscoelastic blood flow model, Comput. Methods Biomech. Biomed. Engrg., 18 (2015), pp. 1704-1725.

[25] X. Wang, S. Nishi, M. Matsukawa, A. Ghigo, P.-Y. Lagrée, and J.-M. Fullana, Fluid friction and wall viscosity of the $1 d$ blood flow model, J. Biomechanics, 49 (2016), pp. 565-571.

[26] M. Zagzoule and J.-P. Marc-Vergnes, A global mathematical model of the cerebral circulation in man, J. Biomechanics, 19 (1986), pp. 1015-1022.

[27] Y. Zocalo, D. Bia, R. Lluberas, and R. L. Armentano, Verification and comparaison of four numerical schemes for a $1 d$ viscoelastic blood flow model, Biological Research, 41 (2008), pp. 227-233. 
DEBAYAN MAITY

TifR Centre for Applicable Mathematics,

560065 BANGAlORE, KARNATAKA, India.

Email address: debayan@tifrbng.res.in

JEAN-PiERRE RAYMOND

Université de Toulouse \& CNRS, UPS, Institut de Mathématiques,

31062 Toulouse Cedex 9, France.

Email address: raymond@math.univ-toulouse.fr

ARnAB RoY

Institute of Mathematics of the Czech Academy of Sciences,

Žitná 25, 11567 Praha 1, Czech Republic.

Email address: royarnab244@gmail.com 